Wildlife Use of NPDES Outfalls

at Los Alamos National Laboratory

Teralene Foxx

Brenda Blea-Edeskuty 



\section{DISCLAIMER}

Portions of this document may be illegible in electronic image products. Images are produced from the best available original document. 


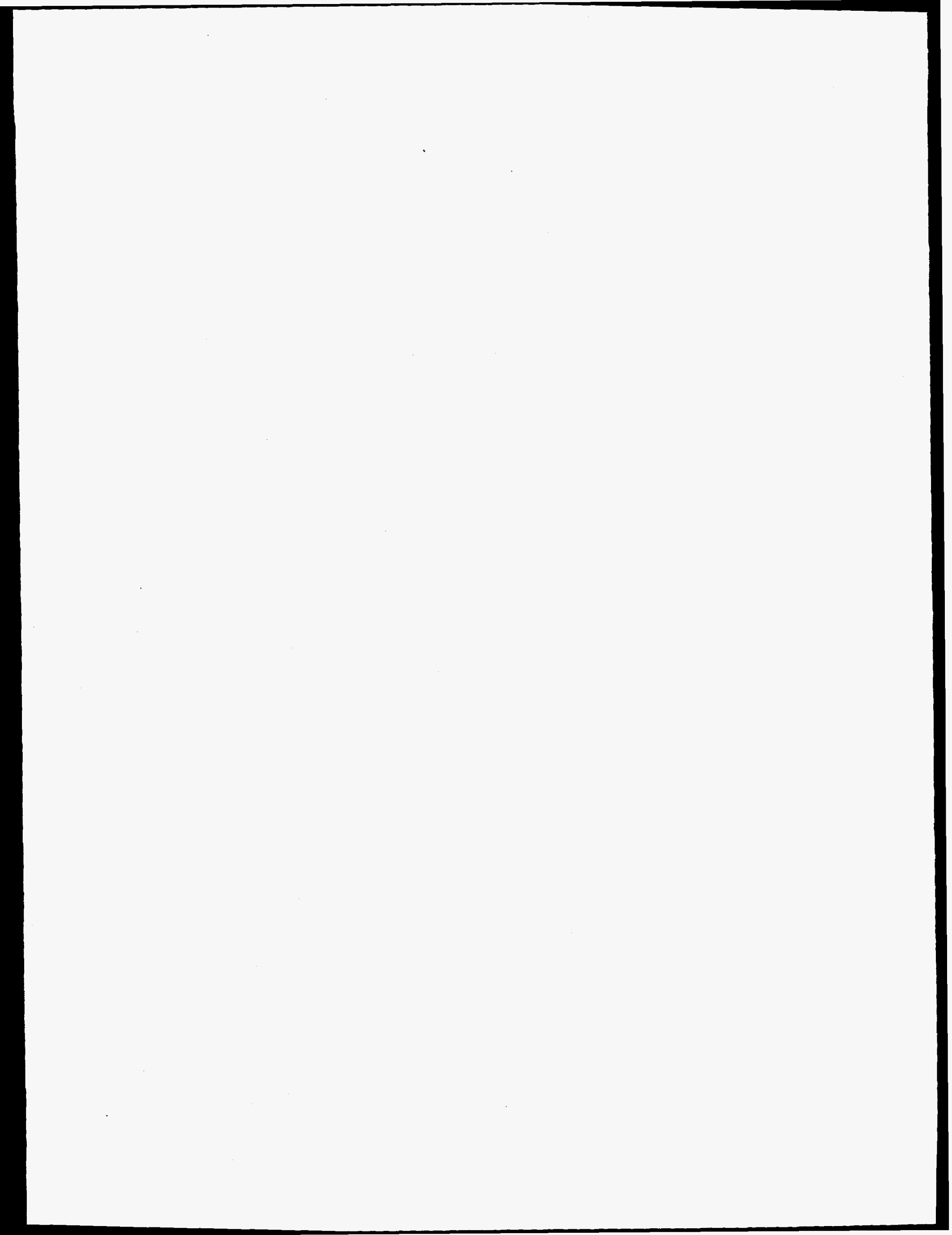




\section{Table of Contents}

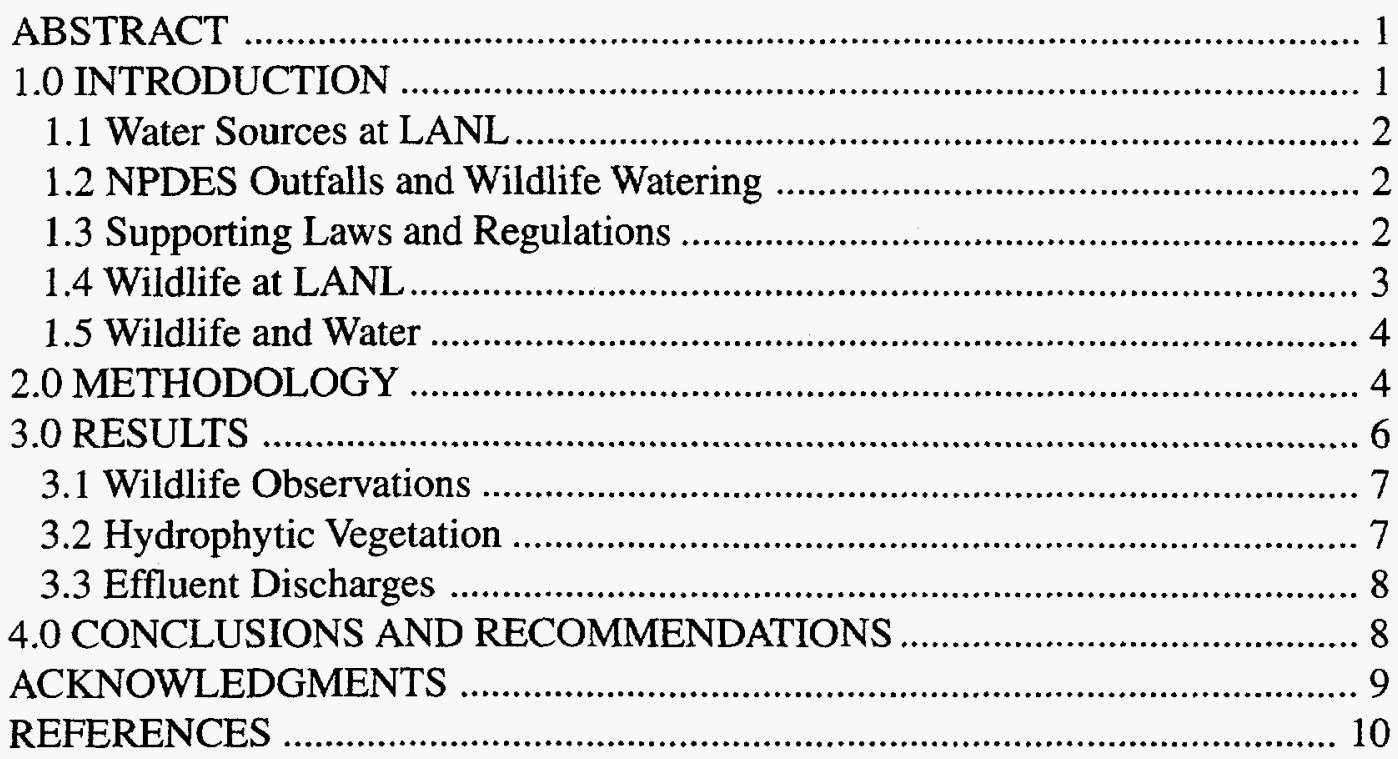




\title{
WILDLIFE USE OF NPDES OUTFALLS AT LOS ALAMOS NATIONAL LABORATORY
}

by

Teralene Foxx and Brenda Blea-Edeskuty

\begin{abstract}
From July through October of 1991, the Biological Resources Evaluation Team (BRET) surveyed 133 of the 140 National Pollutant Discharge and Elimination System outfalls at Los Alamos National Laboratory (LANL). The purpose of the survey was to determine the use of these wastewater outfalls by wildlife. BRET observed wildlife or evidence of wildlife (scat, tracks, or bedding) by 35 vertebrate species in the vicinity of the outfalls, suggesting these animals could be using water from outfalls. Approximately $56 \%$ of the outfalls are probably used or are suitable for use by large mammals as sources of drinking water. Additionally, hydrophytic vegetation grows in association with approximately $40 \%$ of the outfalls-a characteristic that could make these areas eligible for wetland status.

BRET recommends further study to accurately characterize the use of outfalls by small and medium-sized mammals and amphibians. The team also recommends systematic aquatic macroinvertebrate studies to provide information on resident communities and water quality. Wetland assessments may be necessary to ensure compliance with wetland regulations if LANL activities affect any of the outfalls supporting hydrophytic vegetation.
\end{abstract}

\subsection{INTRODUCTION}

In 1991, 140 outfalls were approved for discharge throughout Los Alamos National Laboratory (LANL) lands. All of these outfalls are permitted by the United States Environmental Protection Agency (EPA) under the National Pollutant Discharge and Elimination System (NPDES) program. While the Laboratory has compiled considerable information on water quality at these outfalls, little information is available about the use of outfall effluents by wildlife and other organisms. As an initial reconnaissance study, the Biological Resource Evaluation Team (BRET) at LANL surveyed NPDES outfalls for evidence of wildlife. The Water Quality and Toxics section of group ESH-8 at LANL provided the funding for the survey. 


\subsection{Water Sources at LANL}

There are two general types of water sources within the boundaries of LANLnatural and artificial. Natural sources include springs, ephemeral streams, short stretches of perennial streams, and small palustrine wetlands (NWI 1990). Artificial water sources result from effluent discharges at some of the 140 NPDES outfalls. These discharges include sanitary wastewater, power plant discharge, boiler blowdown, treated cooling water, noncontact cooling water, high explosive wastewater, photographic rinsewater, printed circuit board wastewater, and treated industrial wastewater (Table 1). The volume from these outfalls varies from a trickle to over 757000 liters/day (200 000 gallons/day). Several outfalls produce a constant water source, while others discharge intermittently. Most of the outfalls normally discharge into dry arroyos (LANL 1993) and some may provide significant water resources to wildlife in an otherwise dry environment.

\subsection{NPDES Outfalls and Wildlife Watering}

Prior to this study, there had been no intensive surveys of the biota associated with LANL wastewater outfalls. This survey took place between July and October 1991. It was a preliminary survey with the following major goals:

- to determine the use of the outfalls by wildlife

- to document the presence/absence of hydrophytic vegetation downstream from each outfall source

- to determine the presence and distance of surface water flow

\subsection{Supporting Laws and Regulations}

The Clean Water Act (33 U.S.C. 1251 et seq.) protects the quality of the "waters of the United States." Section 402 of the Act establishes the NPDES Permit Program. Under the NPDES program, any entity responsible for the discharge of a pollutant or pollutants into waters of the U.S. (note: this is terminology used in the Act and in the field) from any point source must apply for a permit. LANL is authorized to discharge under NPDES Permit No. NM0028355 for the main technical areas and NPDES Permit No. NM0028576 for the Fenton Hill geothermal site. The EPA regulations for the NPDES Permit Program are found in 40 CFR 122. 
The EPA, Region 6, issues and enforces the Laboratory's NPDES permits. The New Mexico Environment Department (NMED) certifies the permit to meet state requirements, including the Water Quality Standards for Interstate and Intrastate Streams in New Mexico. The permit includes monitoring conditions and effluent limits based on best available technology (BAT) and water quality standards to protect the existing, designated, or attainable uses of the receiving waters.

The New Mexico Water Quality Standards for Interstate and Intrastate Streams (Water Quality Control Commission, 1991) are consistent with Section 101(a)(2) of the Federal Clean Water Act. This section makes clean water a national priority and calls for the preservation and restoration of water sources so they will provide for the "protection and propagation of fish, shellfish, and wildlife...." The ephemeral streams into which the Laboratory discharges have not been designated in Part 2 of the Water Quality Standards for Interstate and Intrastate Streams in New Mexico. The only known existing and attainable use of these ephemeral receiving streams is wildlife watering.

\subsection{Wildlife at LANL}

A variety of definitions for "wildlife" exist. The Wildlife Coordination Act (16 U.S.C. 661-66c) defines wildlife as "birds, fishes, mammals, and all other classes of wild animals." Wildlife management texts regard wildlife as including "free ranging vertebrates in their naturally associated environments " (Bailey 1984). The New Mexico Game and Fish Department (NMGFD) defines wildlife as "those species of fauna that are not human, that have not been domesticated, and [were] not formerly domesticated..." (NMGFD 1978).

For the purposes of this study, BRET used deer and elk as general indicators of wildlife use at outfalls. This is consistent with the traditional and NMGFD definition of wildlife, but should not be construed to indicate that other species are unworthy of consideration. Small- and medium-sized mammals may be more dependent on outfalls than large mammals and may be most severely affected when effluents are polluted or eliminated (Raymer 1994). In addition, amphibians (Heyer 1994) and aquatic macroinvertebrates (EPA 1990) are valuable indicators of water and habitat quality. In future studies, this report's narrow definition of wildlife should be expanded to include other groups of organisms. 


\subsection{Wildlife and Water}

In the semi-arid climates of the southwestern United States, water and the vegetation that grows nearby provide wildlife with drinking water, shelter, and food. Ribbons of riparian vegetation are also used as travel corridors through the arid landscape.

Riparian areas generally support more diverse plant and animal communities than adjacent uplands and sites without water. Riparian vegetation depends on perennial or ephemeral water flow at or near the soil surface and can develop around both natural and artificial water sources.

Wetlands are usually described in terms of hydrology, vegetation, and soils. For example, the Corps of Engineers defines wetlands as "Those areas that are inundated or saturated by surface or groundwater at a frequency and duration sufficient to support, and that under normal circumstances do support, a prevalence of vegetation typically adapted for life in saturated soil conditions" (Federal Register 1982). In some cases, protection of wetlands is mandated by law whether they are sustained by natural or artificial water sources. This reconnaissance study did not comprehensively assess wetland areas and is insufficient to establish their qualifications as jurisdictional wetlands.

In the southwestern United States, wetland and riparian areas make up less that $1 \%$ of the landscape, yet they are the richest environments in terms of biological productivity and diversity (State of Arizona 1990). In the state of Arizona, for example, $75 \%$ of all native wildlife species depend on healthy riparian systems during some part of their life cycle (State of Arizona 1990). In the semi-arid habitats of the LANL area, any source of water has the potential to be useful to wildlife.

\subsection{METHODOLOGY}

The Water Quality and Toxics Section of the Environmental Protection Group (ESH-8) supplied a list of NPDES outfalls at LANL. Appendices A and B list NPDES outfall numbers and corresponding technical areas. All outfall groupings are noted in Table 1. BRET visited 133 of 142 outfalls from July through October of 1991; the remaining nine outfalls infrequently discharged effluents and were not surveyed. Two outfalls, 
EPA-04A-102 and EPA-04A-103, which BRET later discovered had been deleted from the permit, were surveyed and included in these results. The team photographed each outfall's identification marker, discharge pipe, associated channel, and other notable features, while recording information on the following aspects of the site:

- Physical Features of Outfall

Type of discharge pipe (metal, ceramic, PVC, etc.)

Approximate outside diameter of pipe.

(Information on physical features was not collected until several outfalls had been surveyed and researchers realized the diversity of types and sizes of discharge pipes.)

- Physical Features of Effluent

Flow (gallons per minute)

Color

Odor

Presence of suspended particles

In some cases, the team estimated effluent flow rate by timing how long it took to fill a five gallon bucket to the one- or five-gallon mark and converting the rate to gallons per minute (gpm). However, it was not possible to measure the flow rate in this manner at most outfalls. In some cases, the team made a rough estimate of flow; at other outfalls, BRET was able to use estimates of effluent volume provided by Johnson Controls Incorporated personnel.

- Topographical Features

Topography (mesa top, slope, canyon floor, etc.) at the discharge point for each outfall. (In addition, the team walked downstream from each outfall to see if the effluent reached a receiving canyon or watercourse and to record the point where the water went subsurface.)

- Vegetation Type

The dominant overstory and understory vegetation type at the discharge point of each outfall.

To gather data on wildlife use, BRET searched the outfall area for wildlife, tracks, scat, and other animal sign. Large mammal activity and the presence of hydrophytic vegetation were used as primary criteria to categorize the outfalls; in general, the team took the presence of deer or elk and hydrophytic vegetation as an indication of probable wildlife use. The team assigned each outfall to one of the categories summarized as follows: 
- Category 1 (Probable Use): Outfalls used or probably used by wildlife as water sources

Direct wildlife observation, either by the BRET team or by technical area personnel Sightings during previous BRET studies

The presence of hydrophytic vegetation and water pools

- Category 2 (Potential Use): Outfalls that wildlife could use as water sources

Evidence of wildlife use through scat, bedding, tracks, game trails (particularly deer and elk)

Standing water

Long channels with water

Presence of hydrophytic vegetation

- Category 3 (No Significant Use): Outfalls with little or no evidence of wildlife use

Infrequent or lack of discharge from the outfall

Inaccessibility to wildlife due to topography or Laboratory developments and/or fences

\subsection{RESULTS}

Tables 2-8 list the outfalls along with their assigned category of use. The tables also indicate the presence of associated hydrophytic vegetation, deer and elk activity, and game trails. A total of 75 (approximately 56\%) outfalls fell into the probable use or potential use category. Of these 75, 19 (approximately 14\%) fell into the probable use category. Most of the outfalls assigned to Categories 1 and 2 were type O5A (highexplosives wastewater) and SSS (sanitary wastewater) outfalls, and over $70 \%$ of these outfalls were probably used or could be used by wildlife (Table 9). The high incidence of use for these outfalls is probably due to the location of these outfalls in technical areas that are not highly developed and bordered by undeveloped areas.

There were outfalls placed in Category 3 (No Significant Use) that were not flowing at the time of the survey. Many of these outfalls were inactive or discharged infrequently according to site personnel and were placed in Category 3 based on this information. Outfalls determined to be active, however, should be resurveyed during discharge to determine the potential, if any, for wildlife use. A few outfalls which supported hydrophytic vegetation were placed in Category 3 based on inaccessibility of these outfalls to large mammals. It is possible, however, that these outfalls may be used by small mammals or birds. 
Only a small patch of one outfall (EPA-04A-91) in Category 3 (No Significant Use) showed deer or elk sign and hydrophytic vegetation. Field observations indicated that the hydrophytic vegetation at this outfall was probably maintained by storm run-off rather than effluent releases.

\subsection{Wildlife Observations}

BRET identified a variety of wildlife species in association with the outfalls. Tables $10 \mathrm{~A}$ and $10 \mathrm{~B}$ present a summary of the species observed or inferred to visit the outfalls based on tracks, scat, or bedding. Researchers did not directly observe wildlife drinking water from the outfalls, but they did note various passerine birds bathing at EPA-03A-181.

The animal sign most commonly encountered at the outfalls was of deer and elk. BRET found deer sign at $34.6 \%$ of the outfalls and elk sign at $37.6 \%$. Other animal sign commonly encountered was from squirrels $(20.3 \%)$, raccoons $(13.5 \%)$, coyotes $(8.3 \%)$, and rabbits $(7.5 \%)$. BRET observed the following animals within or on the surface of water at various outfalls:

- chorus frog (Pseudacris triseriata)

- larvae of damselflies (Zygoptera), dragonflies (Anisoptera), flies (Diptera), and mayflies (Ephemeroptera)

- scuds (Amphipoda)

- aquatic beetles (Coleoptera)

- water striders (Gerridae)

- whirligig beetles (Gyrinidae)

- midge larvae, "bloodworms" (Chironomidae)

A more thorough search of outfall effluents and more detailed descriptions of their resident communities would provide valuable data that could assist water quality determinations.

\subsection{Hydrophytic Vegetation}

Hydrophytes grow only in moist habitats; they need a consistent water source at or near the soil surface. The presence of hydrophytic vegetation at $40.6 \%$ of all outfalls indicate consistent water flow. 
Hydrophytes may be classified either as facultative or obligate wetland species. Cattails (Typha spp.), coyote willow (Salix exigua), and watercress (Rorippa nasturtiumaquaticum) are obligate wetland species; they grow almost exclusively in wetlands. Willows (Salix spp.), sedges (Carex spp.), cottonwoods (Populus spp.), and barnyard grass (Echinochloa crusgalli), which usually grow in wetlands but sometimes also grow in other habitats, are facultative wetland species. Thus, by noting the vegetation at outfalls (Tables 11A and 11B), BRET was able to identify wetland areas. Miscellaneous observations made while surveying the outfalls are listed in Table 12.

\subsection{Effluent Discharges}

The nature of the receiving channel at each outfall varies. In some cases, effluents flow onto bare ground, while other outfalls discharge into artificial channels or ephemeral or intermittent streams. Lengths of surface flow recorded at some surveyed outfalls are listed in Table 13. The LANL area received above-average rainfall during each month from May through September, 1991 (LANL 1993). At the time of the survey, many streams were running or contained standing water, probably because of the increased precipitation. Because many effluent discharges are also associated with storm runoff, the unusual rainfall probably increased the volumes and distances of surface flow. These problems contributed to uncertainty in measuring lengths of surface flow attributable to outfalls.

\subsection{CONCLUSIONS AND RECOMMENDATIONS}

Effluent discharges at many NPDES outfalls are low in volume and sporadic in nature. In general, outfalls that discharge substantial volumes on a regular basis provide a dependable water source for wildlife and hydrophytic vegetation. Approximately $56 \%$ of all outfalls within LANL boundaries are accessible and probably used by large mammals such as deer and elk.

LANL should initiate wildlife monitoring programs at Category 2 outfalls that may be affected by LANL projects. To provide preconstruction information, we suggest that BRET monitor areas where water volume would decrease or increase due to Laboratory operations. This data would help in the design of mitigation measures and record project impacts. Further monitoring of outfalls would generate baseline data concerning wildlife use. 
Many of the small outfalls that are inaccessible to large mammals may be important to small- and medium-sized mammals. Selected outfalls should be examined to determine the composition and distribution of associated small- and medium-sized mammal communities.

Non-mammals also rely on outfall effluents. Future work is needed to accurately characterize aquatic macroinvertebrates and amphibian communities. Systematic monitoring of these organisms would supplement chemical measurements of effluent water quality.

Hydrophytic vegetation has developed below approximately $40.6 \%$ of the outfalls. If projects are planned in the vicinity, LANL should assess the wetland status of these areas. LANL should also assess the wetland status of hydrophytic vegetation at any outfalls where water flow will be discontinued or substantially decreased. These assessments will ensure compliance with the US Department of Energy (DOE)'s 10 CFR 1022, which outlines the procedures for DOE compliance with Executive Orders 11988 and 11990 protecting flood plains and wetlands. Specific studies on selected wetlands would refine our understanding of wildlife use patterns at NPDES outfalls.

\section{ACKNOWLEDGMENTS}

An extensive project such as this is not accomplished by one or two individuals. The authors wish to acknowledge the support of Steve Rae, group leader of ESH-18. Steve and other personnel within ESH-18 provided necessary funding and background outfall information. We would also like to thank Aletha Banar, Kathy Bennett, Gregory Gray, Steve Jamison, and Delia Raymer for their work in the field conductivity surveys. Additionally, we want to express our thanks to Kathy Bennett, James Biggs, Saul Cross, Eric Pacheco, Delia Raymer, Debbie Risberg, and Don Usner for their help with the compilation and editing of this document. We would especially like to thank Saul Cross for his final shepherding of the paper through to publication. 


\section{REFERENCES}

Bailey, J.A., Principles of Wildlife Management (John Wiley \& Sons, 1984).

United States Department of Energy, 10 CFR 1022, "Compliance with

Floodplain/Wetlands Environmental Review Requirements," pp. 720-727.

Executive Order 11988, "Flooplain Management," President Jimmy Carter (24 May 1977).

Executive Order 11990, "Protection of Wetlands," (1978) as amended by Executive Order 12608 (1987).

Federal Register, "Title 33: Navigation and Navigable Waters; Chapter II, Regulatory Programs of the Corps of Engineers," Vol. 47, No. 138, p 31810 (US Government Printing Office, Washington, DC, 1982).

Heyer, W.R., M.A. Donnelly, R.W. McDiarmid, L.C. Hayek, and M.S. Foster, Measuring and Monitoring Biological Diversity: Standard Methods for Amphibians (Smithsonian Institution Press, 1994).

Los Alamos National Laboratory, Environmental Surveillance at Los Alamos during 1991, Environmental Protection Group, LA-12572-ENV (1993).

National Wetlands Inventory Maps, US Fish and Wildlife Service, prepared for Los Alamos National Laboratory (1990).

New Mexico Game and Fish Department, Comprehensive Plan (State of New Mexico, 1978).

Raymer, D. F., and J. R. Biggs, "Comparison of Small Mammal Species Diversity Near Wastewater Outfalls, Natural Streams, and Dry Canyons," LA-12725-MS (1994), Los Alamos National Laboratory.

Reed, P. B., US Fish and Wildlife Service, "National List of Plant Species That Occur in Wetlands: 1988, New Mexico," NERC-88/18.31 (1988).

State of Arizona, "Final Report and Recommendations of the Governor's Riparian Habitat Task Force," Executive Order 89-16, Streams and Riparian Resources (October 1990).

Water Quality Control Commission, State of New Mexico, "Water Quality Standards for Interstate and Intrastate Streams in New Mexico," (May 29, 1991).

United States Environmental Protection Agency, "Macroinvertebrate Field and Laboratory Methods for Evaluating Biological Condition of Surface Waters," EPA/600/4-90/030 (1990).

United States Fish and Wildlife Service, Environmental Protection Agency, Soil Conservation Service and Army Corps of Engineers, "Federal Manual for Identifying and Delineating Jurisdictional Wetlands." 1989. 
TABLE 1. Water Sources of Outfalls Examined for Wildlife Use

\begin{tabular}{|l|c|}
\hline \multicolumn{1}{|c|}{ WATER SOURCE } & NPDES CATEGORY \\
\hline Sanitary waste water & SSS \\
\hline Power plant discharge & $01 \mathrm{~A}$ \\
\hline Boiler blowdown & $02 \mathrm{~A}$ \\
\hline Treated cooling water & $03 \mathrm{~A}$ \\
\hline Noncontact cooling water & $04 \mathrm{~A}$ \\
\hline High explosive waste water & $05 \mathrm{~A}$ \\
\hline Photographic rinse water & $06 \mathrm{~A}$ \\
\hline Fenton Hill & 001 \\
\hline Printed circuit board & 128 \\
\hline Industrial waste waster trcatment & 051 \\
\hline
\end{tabular}


TABLE 2. Wildlife Watering Potential of Printed Circuit Board (128-128) and Industrial Wastewater Treatment (051-051) and Fenton Hill (001) Outfalls

\begin{tabular}{|l|c|c|c|c|}
\hline OUTFALL & TA & $\mathbf{1}$ & $\mathbf{2}$ & $\mathbf{3}$ \\
\hline $128-128$ & 22 & $\begin{array}{c}* * * \\
\text { DEH }\end{array}$ & & \\
\hline $051-051$ & 50 & & $\begin{array}{c}* * * \\
\mathrm{H}\end{array}$ & \\
\hline $001-001$ & 57 & & & $\begin{array}{c}* * * \\
\mathrm{E}\end{array}$ \\
\hline
\end{tabular}

$$
\begin{array}{ll}
D=D E E R & \mathrm{H}=\text { HYDROPHYTIC VEGETATION } \\
\mathrm{E}=\mathrm{ELK} & \mathrm{G}=\text { GAME TRAILS }
\end{array}
$$

CATEGORY $1=$ Probable use

CATEGORY $2=$ Potential use

CATEGORY $3=$ No significant use

TABLE 3. Wildlife Watering Potential of Power Plant Discharge (01A) and Boiler Blowdown (02A) Outfalls

\begin{tabular}{|l|c|c|c|c|}
\hline OUTFALL & TA & $\mathbf{1}$ & $\mathbf{2}$ & $\mathbf{3}$ \\
\hline $01 \mathrm{~A}-001$ & 03 & $\begin{array}{c}* * * \\
\mathrm{H}\end{array}$ & & \\
\hline $02 \mathrm{~A}-007$ & 16 & $\begin{array}{c}* * * \\
\mathrm{DE}\end{array}$ & & \\
\hline $02 \mathrm{~A}-129$ & 21 & & $* * *$ & \\
\hline
\end{tabular}

$\begin{array}{ll}\mathrm{D}=\text { DEER } & \text { H=HYDROPHYTIC VEGETATION } \\ \mathrm{E}=\text { ELK } & \text { G=GAME TRAILS }\end{array}$

CATEGORY $1=$ Probable use

CATEGORY $2=$ Potential use

CATEGORY $3=$ No significant use 
TABLE 4. Wildlife Watering Potential of Treated Cooling Water Outfalls

CATEGORY

\begin{tabular}{|c|c|c|c|c|}
\hline OUTFALL & TA & 1 & 2 & 3 \\
\hline 03A-009 & 03 & & $\begin{array}{c}* * * \\
\mathrm{H}\end{array}$ & \\
\hline $03 A-020$ & 02 & $\begin{array}{c}* * * \\
D\end{array}$ & & \\
\hline $03 \mathrm{~A}-021$ & 03 & & $\begin{array}{l}* * * \\
\text { DEH }\end{array}$ & \\
\hline 03A-022 & 03 & $\begin{array}{c}* * * * \\
\mathrm{DEH}\end{array}$ & & \\
\hline $03 \mathrm{~A}-023$ & 03 & & & $* * *$ \\
\hline $03 \mathrm{~A}-024$ & 03 & & & $* * *$ \\
\hline $03 \mathrm{~A}-025$ & 03 & & $* * *$ & \\
\hline $03 \mathrm{~A}-027$ & 03 & $\begin{array}{c}* * * * \\
\mathrm{H}\end{array}$ & & \\
\hline $03 \mathrm{~A}-028$ & 15 & & $\begin{array}{l}* * * * \\
\mathrm{EH}\end{array}$ & \\
\hline $03 \mathrm{~A}-031$ & 21 & & $\begin{array}{c}* * * * \\
\mathrm{H}\end{array}$ & \\
\hline $03 \mathrm{~A}-032$ & 21 & & & $* * *$ \\
\hline $03 \mathrm{~A}-034$ & 21 & & $\begin{array}{c}* * * * \\
\mathrm{H} \\
\end{array}$ & \\
\hline $03 \mathrm{~A}-035$ & 21 & & $* * *$ & \\
\hline $03 \mathrm{~A}-036$ & 21 & & $\begin{array}{l}* * * \\
\mathrm{DEH}\end{array}$ & \\
\hline $03 \mathrm{~A}-037$ & 21 & & & $* * *$ \\
\hline $03 \mathrm{~A}-038$ & 33 & & & $\begin{array}{c}* * * \\
\mathrm{H} \\
\end{array}$ \\
\hline $03 \mathrm{~A}-040$ & 43 & & $\begin{array}{c}* * * \\
\mathrm{H} \\
\end{array}$ & \\
\hline$\longdiv { 0 3 A - 0 4 2 }$ & 46 & & $\begin{array}{c}* * \\
\mathrm{H} \\
\end{array}$ & \\
\hline $03 \mathrm{~A}-043$ & 46 & & $* * *$ & \\
\hline $03 \mathrm{~A}-045$ & 48 & & $\begin{array}{c}* * * \\
\mathrm{H} \\
\end{array}$ & \\
\hline $03 \mathrm{~A}-047$ & 53 & & $\begin{array}{l}* * * \\
\mathrm{DH} \\
\end{array}$ & \\
\hline $03 \mathrm{~A}-048$ & 53 & & & $* * *$ \\
\hline $03 \mathrm{~A}-049$ & 53 & & & $* * *$ \\
\hline $03 A-060$ & 16 & & $\begin{array}{l}* * * * \\
\mathrm{DEH} \\
\end{array}$ & \\
\hline $03 \mathrm{~A}-098$ & 59 & & $\begin{array}{c}* * * \\
\mathrm{H} \\
\end{array}$ & \\
\hline $03 \mathrm{~A}-113$ & 53 & & $* * *$ & \\
\hline $03 \mathrm{~A}-114$ & 53 & & $\begin{array}{c}* * * \\
\mathrm{H} \\
\end{array}$ & \\
\hline $03 A-124$ & 46 & & & $\begin{array}{c}* * * * \\
\mathrm{H} \\
\end{array}$ \\
\hline $03 \mathrm{~A}-125$ & 53 & & & $* * *$ \\
\hline
\end{tabular}


CATEGORY

\begin{tabular}{|l|c|c|c|c|}
\hline OUTFALL & TA & $\mathbf{1}$ & $\mathbf{2}$ & $\mathbf{3}$ \\
\hline $03 \mathrm{~A}-130$ & 11 & & $\begin{array}{r}* * * \\
\text { DEG }\end{array}$ & \\
\hline $03 \mathrm{~A}-136$ & 46 & & & $* * *$ \\
\hline $03 \mathrm{~A}-145$ & 53 & & & $* * *$ \\
\hline $03 \mathrm{~A}-146$ & 53 & & & $* * *$ \\
\hline $03 \mathrm{~A}-148$ & 03 & & & $* * *$ \\
\hline $03 \mathrm{~A}-158$ & 21 & & & $* * *$ \\
\hline $03 \mathrm{~A}-160$ & 35 & & & $* * *$ \\
\hline $03 \mathrm{~A}-181$ & 55 & & $\begin{array}{r}* * * \\
\text { DEH }\end{array}$ & \\
\hline
\end{tabular}

$D=D E E R$

H=HYDROPHYTIC VEGETATION

$E=E L K$ $\mathrm{G}=$ GAME TRAILS

CATEGORY $1=$ Probable use

CATEGORY $2=$ Potential use

CATEGORY $3=$ No significant use 
TABLE 5. Wildlife Watering Potential of Non-contact Cooling Water Outfalls

\begin{tabular}{|c|c|c|c|c|}
\hline & & & ATEGO & \\
\hline OUTFALL & TA & 1 & 2 & 3 \\
\hline $04 \mathrm{~A}-013$ & 46 & & SURV耳 & \\
\hline $04 \mathrm{~A}-014$ & 46 & & & **** \\
\hline $04 \mathrm{~A}-016$ & 48 & & & $\begin{array}{c}* * * \\
\mathrm{H} \\
\end{array}$ \\
\hline 04A-018 & 46 & & & $\begin{array}{c}* * * \\
\mathrm{H}\end{array}$ \\
\hline $04 \mathrm{~A}-070$ & 16 & & $\begin{array}{l}* * * \\
D E\end{array}$ & \\
\hline $04 \mathrm{~A}-083$ & 16 & & $\begin{array}{l}* * * * \\
\mathrm{HD}\end{array}$ & \\
\hline 04A-091 & 16 & & & $\begin{array}{c}* * * ; \\
\mathrm{DEH}\end{array}$ \\
\hline $04 \mathrm{~A}-092$ & 16 & & $\begin{array}{c}* * * * \\
\text { DEGH }\end{array}$ & \\
\hline 04A-093 & 15 & $\begin{array}{c}* * * \\
\mathrm{DEH} \\
\end{array}$ & & \\
\hline 04A-094 & 03 & & $\begin{array}{c}* * * * \\
\mathrm{H}\end{array}$ & \\
\hline $04 \mathrm{~A}-101$ & 40 & & & $* * *$ \\
\hline $04 A-102$ & 15 & & & $\begin{array}{l}* * * \\
\mathrm{E}\end{array}$ \\
\hline $04 A-103$ & 15 & & $\begin{array}{c}* * * \\
\mathrm{E} \\
\end{array}$ & \\
\hline $04 \mathrm{~A}-109$ & 03 & $\begin{array}{c}* * * * \\
E\end{array}$ & & \\
\hline $04 A-115$ & 08 & & $\begin{array}{l}* * * \\
\mathrm{EH}\end{array}$ & \\
\hline $04 \mathrm{~A}-117$ & 46 & & & $* * *$ \\
\hline $04 \mathrm{~A}-118$ & 0 & & SURVE & \\
\hline $04 A-126$ & 48 & $\begin{array}{c}* * * \\
\mathrm{H} \\
\end{array}$ & & \\
\hline $04 \mathrm{~A}-127$ & 35 & & $\begin{array}{c}* * * \\
\mathrm{H}\end{array}$ & \\
\hline $04 \mathrm{~A}-131$ & 48 & & $\begin{array}{c}* * * \\
\mathrm{H}\end{array}$ & \\
\hline $04 \mathrm{~A}-133$ & 53 & & SURV耳 & \\
\hline $04 \mathrm{~A}-135$ & 53 & & & $* * *$ \\
\hline $04 \mathrm{~A}-137$ & 48 & & & $* * *$ \\
\hline $04 \mathrm{~A}-139$ & 15 & & & $* * *$ \\
\hline $04 \mathrm{~A}-140$ & 03 & & $\begin{array}{c}* * * * \\
\mathrm{H} \\
\end{array}$ & \\
\hline $04 \mathrm{~A}-141$ & 39 & & & $* * *$ \\
\hline $04 \mathrm{~A}-142$ & 21 & & $\begin{array}{c}* * * * \\
\mathrm{D}\end{array}$ & \\
\hline $04 \mathrm{~A}-143$ & 15 & & $\begin{array}{l}* * * \\
\mathrm{DE}\end{array}$ & \\
\hline
\end{tabular}


CATEGORY

\begin{tabular}{|c|c|c|c|c|}
\hline OUTFALLL & $\mathrm{TA}$ & 1 & 2 & 3 \\
\hline $04 \mathrm{~A}-147$ & 33 & & $\begin{array}{c}* * * * \\
\text { DEH }\end{array}$ & \\
\hline $04 \mathrm{~A}-151$ & 03 & $* * *$ & & \\
\hline $04 \mathrm{~A}-152$ & 48 & & $\begin{array}{c}* * * \\
\mathrm{H}\end{array}$ & \\
\hline $04 \mathrm{~A}-153$ & 48 & & $\begin{array}{c}* * * * \\
\mathrm{H} \\
\end{array}$ & \\
\hline $04 A-155$ & 09 & & & $\begin{array}{l}* * * * \\
D E\end{array}$ \\
\hline $04 \mathrm{~A}-156$ & 39 & & & $\begin{array}{c}* * * \\
\mathrm{E}\end{array}$ \\
\hline $04 \mathrm{~A}-157$ & 16 & $\begin{array}{l}* * * * \\
\text { DEH } \\
\end{array}$ & & \\
\hline $04 A-161$ & 0 & & SURVI & \\
\hline $04 \mathrm{~A}-163$ & 0 & & & $* * *$ \\
\hline $04 \mathrm{~A}-164$ & 0 & & & $* * *$ \\
\hline $04 \mathrm{~A}-165$ & 0 & & & $\begin{array}{c}* * * \\
\mathrm{D}\end{array}$ \\
\hline $04 \mathrm{~A}-166$ & 0 & & SURVE & \\
\hline $04 \mathrm{~A}-167$ & 0 & & SURVE & \\
\hline $04 \mathrm{~A}-168$ & 0 & & & $* * *$ \\
\hline $04 \mathrm{~A}-169$ & 0 & & & $* * *$ \\
\hline $04 \mathrm{~A}-170$ & 0 & & & **** \\
\hline $04 \mathrm{~A}-171$ & 0 & & & $* * *$ \\
\hline $04 \mathrm{~A}-172$ & 0 & & & $* * *$ \\
\hline $04 \mathrm{~A}-173$ & 0 & & & $* * *$ \\
\hline $04 \mathrm{~A}-174$ & 0 & & & $* * *$ \\
\hline $04 \mathrm{~A}-175$ & 0 & & & $* * *$ \\
\hline $04 \mathrm{~A}-176$ & 0 & & & $* * *$ \\
\hline $04 \mathrm{~A}-177$ & 0 & \multicolumn{3}{|c|}{ NOT SURV本YED } \\
\hline $04 \mathrm{~A}-178$ & 0 & & & $* * *$ \\
\hline $04 \mathrm{~A}-179$ & 0 & \multicolumn{3}{|c|}{ NOT SURVEYED } \\
\hline $04 \mathrm{~A}-182$ & 21 & & & $* * *$ \\
\hline
\end{tabular}

$D=D E E R$

H=HYDROPHYTIC VEGETATION

$E=E L K$

$\mathrm{G}=\mathrm{GAME}$ TRAILS

CATEGORY $1=$ Probable use

CATEGORY $2=$ Potential use

CATEGORY $3=$ No significant use 
TABLE 6. Wildlife Watering Potential of High Explosive Waste Water Outfalls

CATEGORY

\begin{tabular}{|c|c|c|c|c|}
\hline OUTFALL & TA & 1 & 2 & 3 \\
\hline $05 \mathrm{~A}-052$ & 16 & & & $\begin{array}{l}* * * \\
\mathrm{DE}\end{array}$ \\
\hline $05 \mathrm{~A}-053$ & 16 & & $\begin{array}{l}* * * \\
\mathrm{DH}\end{array}$ & \\
\hline $05 \mathrm{~A}-054$ & 16 & $\begin{array}{c}* * * \\
\mathrm{DEGH}\end{array}$ & & \\
\hline $05 \mathrm{~A}-055$ & 16 & & $\begin{array}{l}* * * * * \\
\mathrm{G}\end{array}$ & \\
\hline $05 \mathrm{~A}-056$ & 16 & & $\begin{array}{l}* * * * * \\
\mathrm{DE}\end{array}$ & \\
\hline $05 A-057$ & 16 & & & $\begin{array}{l}* * * * * \\
\mathrm{DE}\end{array}$ \\
\hline 05A-058 & 16 & $\begin{array}{c}* * * * \\
D E H \\
\end{array}$ & & \\
\hline $05 \mathrm{~A}-061$ & 16 & & $\begin{array}{c}* * * \\
\mathrm{DEH} \\
\end{array}$ & \\
\hline $05 \mathrm{~A}-062$ & 16 & & & $\begin{array}{c}* * * \\
\mathrm{D} \\
\end{array}$ \\
\hline $05 A-063$ & 16 & & & $\begin{array}{c}* * * \\
\mathrm{DEH} \\
\end{array}$ \\
\hline $05 A-066$ & 09 & & $\begin{array}{c}* * * \\
\text { DEH } \\
\end{array}$ & \\
\hline $05 \mathrm{~A}-067$ & 09 & & $\begin{array}{c}* * * * \\
\mathrm{E} \\
\end{array}$ & \\
\hline $05 A-068$ & 09 & & $\begin{array}{l}* * * \\
\text { EG }\end{array}$ & \\
\hline $05 \mathrm{~A}-069$ & 11 & & $\begin{array}{l}* * * \\
\mathrm{DE}\end{array}$ & \\
\hline $05 \mathrm{~A}-071$ & 16 & & $\begin{array}{c}* * * \\
\text { DEH } \\
\end{array}$ & \\
\hline $05 \mathrm{~A}-072$ & 16 & $\begin{array}{c}* * * \\
\text { DEH }\end{array}$ & & \\
\hline 05A-096 & 11 & & $\begin{array}{c}* * * \\
\text { DEG } \\
\end{array}$ & \\
\hline $05 \mathrm{~A}-097$ & 11 & & $\begin{array}{c}* * * * \\
\mathrm{D} \\
\end{array}$ & \\
\hline $05 A-149$ & 16 & & & $\begin{array}{l}* * * \\
\mathrm{DE} \\
\end{array}$ \\
\hline $05 \mathrm{~A}-154$ & 40 & & & $* * *$ \\
\hline $05 A-159$ & 16 & & $\begin{array}{l}* * * \\
\mathrm{DE}\end{array}$ & \\
\hline
\end{tabular}

$D=D E E R$

H=HYDROPHYTIC VEGETATION

$E=E L K$

$\mathrm{G}=\mathrm{GAME}$ TRAILS

CATEGORY $1=$ Probable use

CATEGORY $2=$ Potential use

CATEGORY $3=$ No significant use 
TABLE 7. Wildlife Watering Potential of Photographic Rinsewater Outfalls

\begin{tabular}{|l|c|c|c|c|}
\hline OUTFALI & TA & $\mathbf{1}$ & $\mathbf{2}$ & $\mathbf{3}$ \\
\hline $06 \mathrm{~A}-073$ & 16 & $\begin{array}{r}* * * \\
\text { DH }\end{array}$ & & \\
\hline $06 \mathrm{~A}-074$ & 08 & $\begin{array}{r}* * * \\
\text { EG }\end{array}$ & & \\
\hline $06 \mathrm{~A}-075$ & 08 & $\begin{array}{c}* * * \\
\text { DEH }\end{array}$ & & \\
\hline $06 \mathrm{~A}-078$ & 22 & & $\begin{array}{c}* * * \\
\mathrm{H}\end{array}$ & \\
\hline $06 \mathrm{~A}-079$ & 40 & & & $* * *$ \\
\hline $06 \mathrm{~A}-080$ & 40 & & & $* * *$ \\
\hline $06 \mathrm{~A}-081$ & 40 & & & $* * *$ \\
\hline $06 \mathrm{~A}-082$ & 40 & & & $* * *$ \\
\hline $06 \mathrm{~A}-099$ & 40 & & $* * *$ & \\
\hline $06 \mathrm{~A}-100$ & 40 & & & $* * *$ \\
\hline $06 \mathrm{~A}-106$ & 36 & & & $* * *$ \\
\hline $06 \mathrm{~A}-123$ & 15 & & $* * *$ & \\
\hline $06 \mathrm{~A}-132$ & 35 & & & $* * *$ \\
\hline
\end{tabular}

$\mathrm{D}=\mathrm{DEER}$

H=HYDROPHYTIC VEGETATION

$\mathrm{E}=\mathrm{ELK}$

$\mathrm{G}=\mathrm{GAME}$ TRAILS

CATEGORY $1=$ Probable use

CATEGORY $2=$ Potential use

CATEGORY $3=$ No significant use 
TABLE 8. Wildlife Watering Potential of Sanitary Wastewater Outfalls

\begin{tabular}{|l|c|c|c|c|}
\hline \multicolumn{1}{c|}{ CATEGORY } \\
\hline OUTFALL & TA & 1 & 2 & 3 \\
\hline SSS-01S & 03 & $\begin{array}{r}* * * \\
\text { EH }\end{array}$ & & \\
\hline SSS-02S & 09 & & $\begin{array}{r}* * * \\
\text { E }\end{array}$ & \\
\hline SSS-03S & 16 & & $\begin{array}{r}* * * \\
\text { DE }\end{array}$ & \\
\hline SSS-04S & 18 & $* * *$ & & \\
\hline SSS-05S & 21 & & $* * *$ & \\
\hline SSS-06S & 41 & & $* * *$ & \\
\hline SSS-07S & 46 & & & $* * *$ \\
\hline SSS-09S & 53 & & & $* * *$ \\
\hline SSS-10S & 35 & & $* * *$ & \\
\hline SSS-12S & 46 & & & $* * *$ \\
\hline SSS-13S & 46 & & NOT SURVEY & \\
\hline
\end{tabular}

$\begin{array}{ll}\text { D=DEER } & \text { H=HYDROPHYTIC VEGETATION } \\ \text { E=ELK } & \text { G=GAME TRAILS }\end{array}$

CATEGORY $1=$ Probable use

CATEGORY $2=$ Potential use

CATEGORY $3=$ No significant use 
TABLE 9. Percent of Outfalls within each Category of Use (Outfalls not surveyed are not included.)

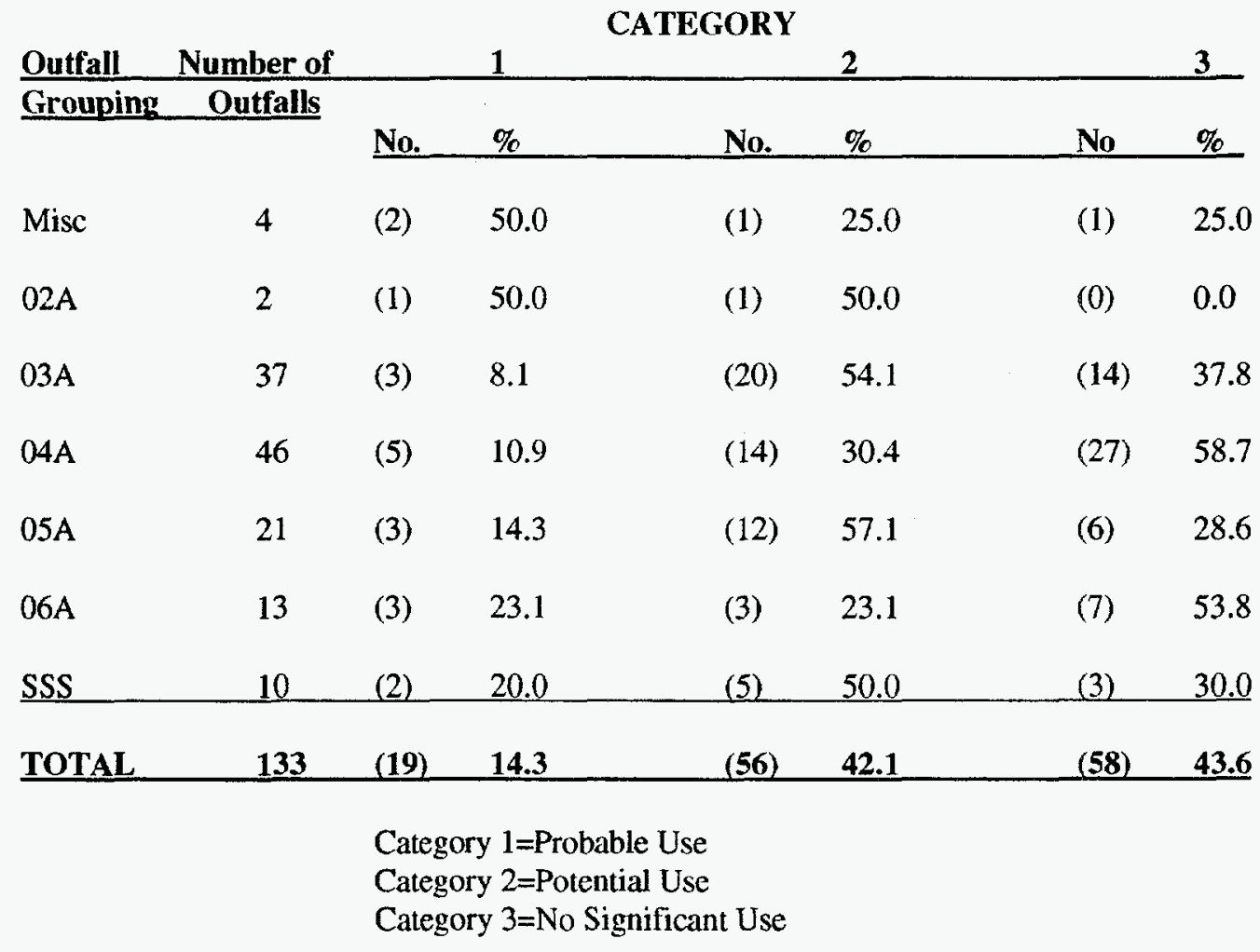


Table 10A. Fauna Associated with Outfalls

\begin{tabular}{|c|c|c|c|}
\hline COMMON NAME & SCIENTIFIC NAME & OUTFALL NO & TA \\
\hline ABERT'S SQUIRREL & Sciurus aberti & $\begin{array}{l}\text { EPA-05A-054 } \\
\text { EPA-128-128 }\end{array}$ & $\begin{array}{l}\text { TA-16 } \\
\text { TA-22 }\end{array}$ \\
\hline AQUATIC BEETLE & Coleoptera & $\begin{array}{l}\text { EPA-03A-020 } \\
\text { EPA-03A-021 } \\
\text { EPA-06A-075 } \\
\text { EPA-128-128 }\end{array}$ & $\begin{array}{l}\text { TA-02 } \\
\text { TA-03 } \\
\text { TA-08 } \\
\text { TA-22 } \\
\end{array}$ \\
\hline BEAR & Ursus americanus & $\begin{array}{l}\text { EPA-03A-130 } \\
\text { EPA-05A-069 } \\
\text { EPA-05A-096 } \\
\text { EPA-06A-080 }\end{array}$ & $\begin{array}{l}\text { TA-11 } \\
\text { TA-11 } \\
\text { TA-11 } \\
\text { TA-40 }\end{array}$ \\
\hline CHICKADEE & Parus sp. & $\begin{array}{l}\text { EPA-03A-020 } \\
\text { EPA-128-128 }\end{array}$ & $\begin{array}{l}\text { TA-02 } \\
\text { TA-22 }\end{array}$ \\
\hline BLOODWORM & Chironomidae & $\begin{array}{l}\text { EPA-03A-021 } \\
\text { EPA-03A-025 }\end{array}$ & $\begin{array}{l}\text { TA-03 } \\
\text { TA-03 }\end{array}$ \\
\hline$\overline{\mathrm{BOBCAT}}$ & Felis rufus & EPA-128-128 & TA-22 \\
\hline CHIPMUNK & Eutamias sp. & $\begin{array}{l}\text { EPA-03A-020 } \\
\text { EPA-03A-045 } \\
\text { EPA-03A-114 } \\
\text { EPA-04A-109 } \\
\text { EPA-05A-063 }\end{array}$ & $\begin{array}{l}\text { TA-02 } \\
\text { TA-48 } \\
\text { TA-53 } \\
\text { TA-03 } \\
\text { TA-16 }\end{array}$ \\
\hline CHORUS FROG & Pseudacris triseriata & EPA-06A-073 & TA-16 \\
\hline COYOTE & Canis latrans & $\begin{array}{l}\text { EPA-02A-007 } \\
\text { EPA-03A-020 } \\
\text { EPA-03A-038 } \\
\text { EPA-04A-143 } \\
\text { EPA-04A-157 } \\
\text { EPA-05A-067 } \\
\text { EPA-05A-096 } \\
\text { EPA-06A-075 } \\
\text { EPA-128-128 } \\
\text { EPA-SSS-01S } \\
\text { EPA-SSS-02S }\end{array}$ & $\begin{array}{l}\text { TA-16 } \\
\text { TA-02 } \\
\text { TA-33 } \\
\text { TA-15 } \\
\text { TA-16 } \\
\text { TA-09 } \\
\text { TA-11 } \\
\text { TA-08 } \\
\text { TA-22 } \\
\text { TA-03 } \\
\text { TA-09 }\end{array}$ \\
\hline DAMSELFLY & Zygoptera & $\begin{array}{l}\text { EPA-03A-023 } \\
\text { EPA-04A-016 } \\
\text { EPA-04A-094 } \\
\text { EPA-04A-109 } \\
\text { EPA-04A-115 } \\
\text { EPA-04A-152 } \\
\text { EPA-06A-074 } \\
\text { EPA-SSS-03S } \\
\text { EPA-SSS-04S }\end{array}$ & $\begin{array}{l}\text { TA-03 } \\
\text { TA-48 } \\
\text { TA-03 } \\
\text { TA-03 } \\
\text { TA-08 } \\
\text { TA-48 } \\
\text { TA-08 } \\
\text { TA-16 } \\
\text { TA-18 }\end{array}$ \\
\hline DAMSELFLY LARVA & Zygoptera & $\begin{array}{l}\text { EPA-04A-094 } \\
\text { EPA-05A-054 }\end{array}$ & $\begin{array}{l}\text { TA-03 } \\
\text { TA-16 }\end{array}$ \\
\hline DARK-EYED JUNCO & Junco hyemalis & EPA-03A-181 & TA-55 \\
\hline DEER MOUSE & Peromyscus maniculatus & $\begin{array}{l}\text { EPA-03A-020 } \\
\text { EPA-128-128 }\end{array}$ & $\begin{array}{l}\text { TA-02 } \\
\text { TA-22 }\end{array}$ \\
\hline DIPTERAN LARVA & Diptera & $\begin{array}{l}\text { EPA-04A-109 } \\
\text { EPA-05A-054 } \\
\text { EPA-SSS-04S }\end{array}$ & $\begin{array}{l}\text { TA-03 } \\
\text { TA-16 } \\
\text { TA-18 }\end{array}$ \\
\hline$\overline{\text { DRAGONFLY }}$ & Anisoptera & $\begin{array}{l}\text { EPA-03A-022 } \\
\text { EPA-03A-042 } \\
\text { EPA-04A-126 } \\
\text { EPA-05A-058 } \\
\text { EPA-SSS-04S }\end{array}$ & $\begin{array}{l}\text { TA-03 } \\
\text { TA-46 } \\
\text { TA-48 } \\
\text { TA-16 } \\
\text { TA-18 }\end{array}$ \\
\hline
\end{tabular}




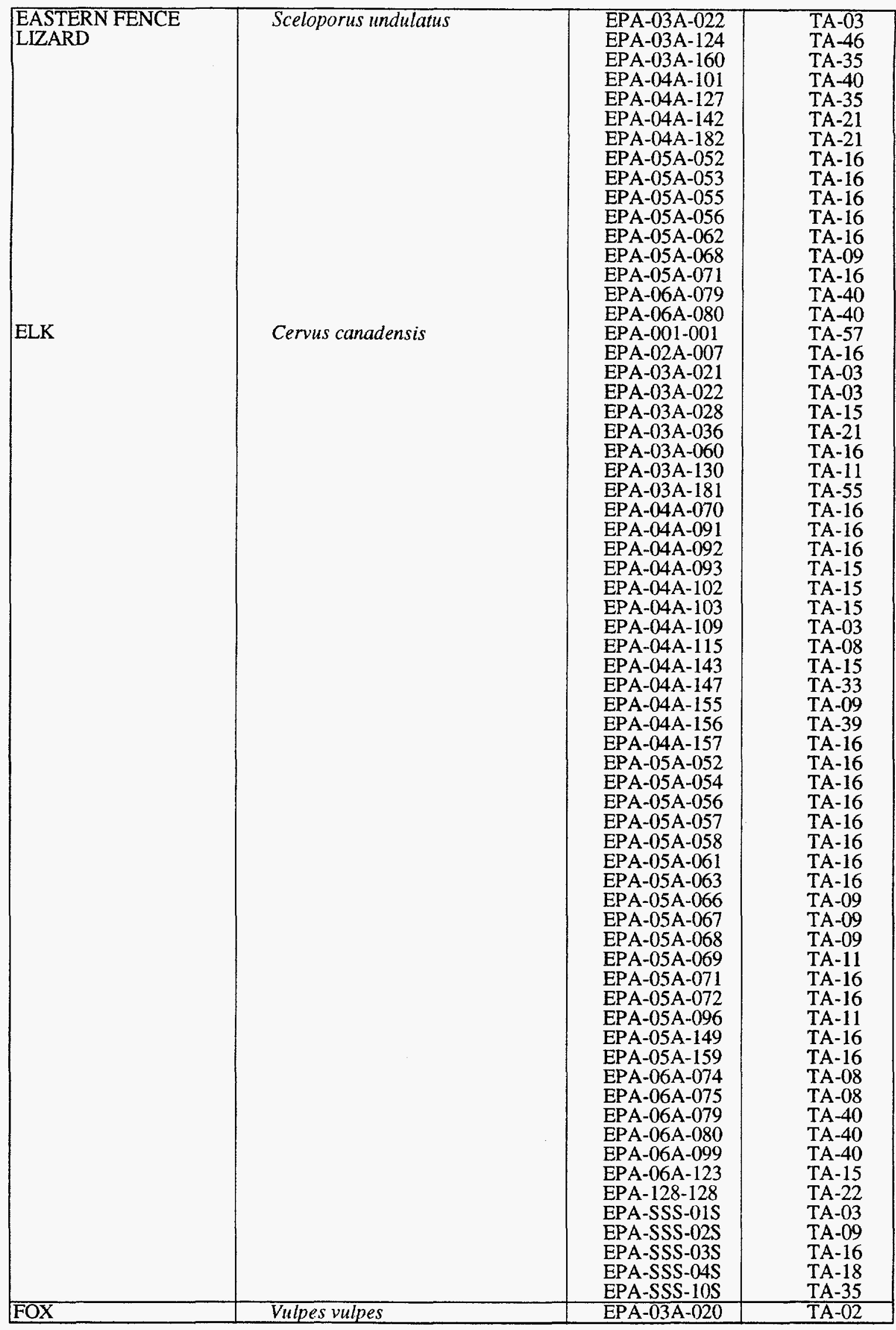




\begin{tabular}{|c|c|c|c|}
\hline GARTER SNAKE & Thamnophis elegans & EPA-03A-040 & $\overline{\mathrm{TA}-43}$ \\
\hline GOPHER & Thomomys talpoides & $\begin{array}{l}\text { EPA-001-001 } \\
\text { EPA-02A-007 } \\
\text { EPA-03A-130 } \\
\text { EPA-04A-102 } \\
\text { EPA-04A-141 } \\
\text { EPA-04A-142 } \\
\text { EPA-04A-156 } \\
\text { EPA-04A-157 } \\
\text { EPA-04A-176 } \\
\text { EPA-04A-182 } \\
\text { EPA-05A-052 } \\
\text { EPA-SSS-04S }\end{array}$ & $\begin{array}{l}\text { TA-57 } \\
\text { TA-16 } \\
\text { TA-11 } \\
\text { TA-15 } \\
\text { TA-39 } \\
\text { TA-21 } \\
\text { TA-39 } \\
\text { TA-16 } \\
\text { TA-0 } \\
\text { TA-21 } \\
\text { TA-16 } \\
\text { TA-18 }\end{array}$ \\
\hline GREAT HORNED OWL & Bubo virginianus & EPA-128-128 & TA-22 \\
\hline ISOPOD & Isopoda & EPA-04A-109 & TA-03 \\
\hline JUNE BEETLE & Scarabaeidae & EPA-03A-022 & TA-03 \\
\hline LINCOLN SPARROW & Melospiza lincolnii & EPA-03A-181 & TA-55 \\
\hline LIZARD & Gekkonidae & EPA-03A-047 & TA-53 \\
\hline MAYFLY LARVA & Ephemeroptera & $\begin{array}{l}\text { EPA-03A-021 } \\
\text { EPA-03A-042 } \\
\text { EPA-04A-152 } \\
\text { EPA-05A-054 } \\
\text { EPA-SSS-04S }\end{array}$ & $\begin{array}{l}\text { TA-03 } \\
\text { TA-46 } \\
\text { TA-48 } \\
\text { TA-16 } \\
\text { TA-18 }\end{array}$ \\
\hline MOSQUTTO LARVA & Culicidae & $\begin{array}{l}\text { EPA-03A-028 } \\
\text { EPA-03A-042 } \\
\text { EPA-03A-043 } \\
\text { EPA-04A-115 } \\
\text { EPA-06A-074 } \\
\text { EPA-06A-075 }\end{array}$ & $\begin{array}{l}\text { TA-15 } \\
\text { TA-46 } \\
\text { TA-46 } \\
\text { TA-08 } \\
\text { TA-08 } \\
\text { TA-08 }\end{array}$ \\
\hline $\begin{array}{l}\text { MOURNING CLOAK } \\
\text { BUTTERFLY }\end{array}$ & Nymphalis antiopa & EPA-03A-020 & TA-02 \\
\hline MOUSE & Cricetidae & EPA-04A-083 & TA-16 \\
\hline \begin{tabular}{|l} 
MULE DEER \\
\end{tabular} & Odocoileus hemionus & $\begin{array}{l}\text { EPA-02A-007 } \\
\text { EPA-03A-020 } \\
\text { EPA-03A-021 } \\
\text { EPA-03A-022 } \\
\text { EPA-03A-036 } \\
\text { EPA-03A-047 } \\
\text { EPA-03A-060 } \\
\text { EPA-03A-130 } \\
\text { EPA-03A-181 } \\
\text { EPA-04A-070 } \\
\text { EPA-04A-083 } \\
\text { EPA-04A-091 } \\
\text { EPA-04A-092 } \\
\text { EPA-04A-093 } \\
\text { EPA-04A-142 } \\
\text { EPA-04A-143 } \\
\text { EPA-04A-147 } \\
\text { EPA-04A-155 } \\
\text { EPA-04A-157 } \\
\text { EPA-04A-165 } \\
\text { EPA-05A-052 } \\
\text { EPA-05A-053 } \\
\text { EPA-05A-054 } \\
\text { EPA-05A-056 } \\
\text { EPA-05A-057 } \\
\text { EPA-05A-058 } \\
\text { EPA-05A-061 } \\
\text { EPA-05A-062 } \\
\text { EPA-05A-063 } \\
\text { EPA-05A-066 } \\
\text { EPA-05A-069 } \\
\text { EPA-05A-071 }\end{array}$ & $\begin{array}{l}\text { TA-16 } \\
\text { TA-02 } \\
\text { TA-03 } \\
\text { TA-03 } \\
\text { TA-21 } \\
\text { TA-53 } \\
\text { TA-16 } \\
\text { TA-11 } \\
\text { TA-55 } \\
\text { TA-16 } \\
\text { TA-16 } \\
\text { TA-16 } \\
\text { TA-16 } \\
\text { TA-15 } \\
\text { TA-21 } \\
\text { TA-15 } \\
\text { TA-33 } \\
\text { TA-09 } \\
\text { TA-16 } \\
\text { TA-0 } \\
\text { TA-16 } \\
\text { TA-16 } \\
\text { TA-16 } \\
\text { TA-16 } \\
\text { TA-16 } \\
\text { TA-15 } \\
\text { TA-16 } \\
\text { TA-16 } \\
\text { TA-16 } \\
\text { TA-09 } \\
\text { TA-11 } \\
\text { TA-16 }\end{array}$ \\
\hline
\end{tabular}




\begin{tabular}{|c|c|c|c|}
\hline $\begin{array}{l}\text { MULEE DEER } \\
\text { CONTINUED }\end{array}$ & & $\begin{array}{l}\text { EPA-05A-072 } \\
\text { EPA-05A-096 } \\
\text { EPA-05A-097 } \\
\text { EPA-05A-149 } \\
\text { EPA-05A-159 } \\
\text { EPA-06A-073 } \\
\text { EPA-06A-075 } \\
\text { EPA-06A-080 } \\
\text { EPA-06A-123 } \\
\text { EPA-128-128 } \\
\text { EPA-SSS-03S } \\
\text { EPA-SSS-04S } \\
\text { EPA-SSS-05S } \\
\text { EPA-SSS-10S }\end{array}$ & $\begin{array}{l}\text { TA-16 } \\
\text { TA-11 } \\
\text { TA-11 } \\
\text { TA-16 } \\
\text { TA-16 } \\
\text { TA-16 } \\
\text { TA-08 } \\
\text { TA-40 } \\
\text { TA-15 } \\
\text { TA-22 } \\
\text { TA-16 } \\
\text { TA-18 } \\
\text { TA-21 } \\
\text { TA-35 }\end{array}$ \\
\hline PINYON JAY & Gymnorhinus cyanocephalus & EPA-04A-147 & TA-33 \\
\hline PORCUPINE & Erethizon dorsatum & $\begin{array}{l}\text { EPA-05A-053 } \\
\text { EPA-05A-054 }\end{array}$ & $\begin{array}{l}\text { TA-16 } \\
\text { TA-16 }\end{array}$ \\
\hline RABBIT & Sylvilagus sp. & $\begin{array}{l}\text { EPA-001-001 } \\
\text { EPA-03A-038 } \\
\text { EPA-03A-114 } \\
\text { EPA-04A-093 } \\
\text { EPA-04A-131 } \\
\text { EPA-04A-140 } \\
\text { EPA-04A-147 } \\
\text { EPA-05A-054 } \\
\text { EPA-05A-058 } \\
\text { EPA-05A-062 }\end{array}$ & $\begin{array}{l}\text { TA-57 } \\
\text { TA-33 } \\
\text { TA-53 } \\
\text { TA-15 } \\
\text { TA-48 } \\
\text { TA-03 } \\
\text { TA-33 } \\
\text { TA-16 } \\
\text { TA-16 } \\
\text { TA-16 }\end{array}$ \\
\hline RACCOON & Procyon lotor & $\begin{array}{l}\text { EPA-03A-020 } \\
\text { EPA-03A-025 } \\
\text { EPA-03A-040 } \\
\text { EPA-03A-098 } \\
\text { EPA-03A-113 } \\
\text { EPA-03A-114 } \\
\text { EPA-04A-109 } \\
\text { EPA-04A-126 } \\
\text { EPA-04A-131 } \\
\text { EPA-04A-137 } \\
\text { EPA-04A-140 } \\
\text { EPA-04A-147 } \\
\text { EPA-04A-151 } \\
\text { EPA-04A-152 } \\
\text { EPA-05A-063 } \\
\text { EPA-128-128 } \\
\text { EPA-SSS-01S } \\
\text { EPA-SSS-04S }\end{array}$ & $\begin{array}{l}\text { TA-02 } \\
\text { TA-03 } \\
\text { TA-43 } \\
\text { TA-59 } \\
\text { TA-53 } \\
\text { TA-53 } \\
\text { TA-03 } \\
\text { TA-48 } \\
\text { TA-48 } \\
\text { TA-48 } \\
\text { TA-03 } \\
\text { TA-33 } \\
\text { TA-03 } \\
\text { TA-48 } \\
\text { TA-16 } \\
\text { TA-22 } \\
\text { TA-03 } \\
\text { TA-18 }\end{array}$ \\
\hline RATTLESNAKE & Crotalinae & EPA-03A-025 & TA-03 \\
\hline RAVEN & Corvus corax & $\begin{array}{l}\text { EPA-03A-181 } \\
\text { EPA-04A-109 } \\
\text { EPA-128-128 }\end{array}$ & $\begin{array}{l}\text { TA-55 } \\
\text { TA-03 } \\
\text { TA-22 }\end{array}$ \\
\hline RED SQUUIRREL & Sciurus arizonensis & EPA-04A-109 & TA-03 \\
\hline ROBIN & Turdus migratorius & EPA-03A-114 & TA-53 \\
\hline $\begin{array}{l}\text { SHORT HORNED } \\
\text { LIZARD }\end{array}$ & Phrynosoma douglassi & EPA-05A-053 & TA-16 \\
\hline SHREW & Sorex sp. & EPA-05A-071 & TA-16 \\
\hline SHRIMP & Amphipoda & EPA-128-128 & TA-22 \\
\hline SKUNK & Mephitis sp. & EPA-05A-063 & TA-16 \\
\hline SNAIL & Gastropoda & EPA-SSS-04S & TA-18 \\
\hline
\end{tabular}




\begin{tabular}{|c|c|c|c|}
\hline SQUIRREL & Spermophilus sp. & $\begin{array}{l}\text { EPA-03A-022 } \\
\text { EPA-03A-034 } \\
\text { EPA-03A-036 } \\
\text { EPA-03A-045 } \\
\text { EPA-03A-048 } \\
\text { EPA-03A-130 } \\
\text { EPA-04A-091 } \\
\text { EPA-04A-092 } \\
\text { EPA-04A-093 } \\
\text { EPA-04A-109 } \\
\text { EPA-04A-127 } \\
\text { EPA-04A-131 } \\
\text { EPA-04A-140 } \\
\text { EPA-04A-157 } \\
\text { EPA-04A-182 } \\
\text { EPA-05A-054 } \\
\text { EPA-05A-055 } \\
\text { EPA-05A-056 } \\
\text { EPA-05A-058 } \\
\text { EPA-05A-062 } \\
\text { EPA-05A-063 } \\
\text { EPA-05A-066 } \\
\text { EPA-05A-096 } \\
\text { EPA-06A-075 } \\
\text { EPA-06A-132 } \\
\text { EPA-SSS-03S } \\
\text { EPA-SSS-05S }\end{array}$ & $\begin{array}{l}\text { TA-03 } \\
\text { TA-21 } \\
\text { TA-21 } \\
\text { TA-48 } \\
\text { TA-53 } \\
\text { TA-11 } \\
\text { TA-16 } \\
\text { TA-16 } \\
\text { TA-15 } \\
\text { TA-03 } \\
\text { TA-35 } \\
\text { TA-48 } \\
\text { TA-03 } \\
\text { TA-16 } \\
\text { TA-21 } \\
\text { TA-16 } \\
\text { TA-16 } \\
\text { TA-16 } \\
\text { TA-16 } \\
\text { TA-16 } \\
\text { TA-16 } \\
\text { TA-09 } \\
\text { TA-11 } \\
\text { TA-08 } \\
\text { TA-35 } \\
\text { TA-16 } \\
\text { TA-21 }\end{array}$ \\
\hline TURKEY VULTURES & Cathartes aura & $\begin{array}{l}\text { EPA-03A-034 } \\
\text { EPA-SSS-09S }\end{array}$ & $\begin{array}{l}\text { TA-21 } \\
\text { TA-53 }\end{array}$ \\
\hline VOLE & Microtus sp. & $\begin{array}{l}\text { EPA-03A-020 } \\
\text { EPA-03A-025 }\end{array}$ & $\begin{array}{l}\text { TA-02 } \\
\text { TA-03 }\end{array}$ \\
\hline WATER STRIDERS & Gerridae & $\begin{array}{l}\text { EPA-04A-157 } \\
\text { EPA-05A-058 } \\
\text { EPA-05A-069 } \\
\text { EPA-06A-073 } \\
\text { EPA-128-128 } \\
\text { EPA-SSS-03S } \\
\text { EPA-SSS-04S }\end{array}$ & $\begin{array}{l}\text { TA-16 } \\
\text { TA-15 } \\
\text { TA-11 } \\
\text { TA-16 } \\
\text { TA-22 } \\
\text { TA-16 } \\
\text { TA-18 }\end{array}$ \\
\hline WEASEL & Mustela sp. & $\begin{array}{l}\text { EPA-03A-027 } \\
\text { EPA-03A-113 } \\
\text { EPA-04A-093 }\end{array}$ & $\begin{array}{l}\text { TA-03 } \\
\text { TA-53 } \\
\text { TA-15 }\end{array}$ \\
\hline WHIPTAIL LIZARD & Cnemidophorus sp. & EPA-03A-113 & TA-53 \\
\hline WHIRLIGIG BEETLE & Gyrinidae & $\begin{array}{l}\text { EPA-04A-094 } \\
\text { EPA-04A-157 } \\
\text { EPA-06A-073 }\end{array}$ & $\begin{array}{l}\text { TA-03 } \\
\text { TA-16 } \\
\text { TA-16 }\end{array}$ \\
\hline $\begin{array}{l}\text { WHITE CROWNED } \\
\text { SPARROW }\end{array}$ & Zonotrichia leucophrys & EPA-03A-181 & TA-55 \\
\hline WOODRAT & Neotoma sp. & EPA-06A-106 & TA-36 \\
\hline
\end{tabular}


Table 10B. Fauna Associated with Outfalls.

\begin{tabular}{|c|c|c|c|}
\hline OUTFALL NO. & TA & COMMON NAME & SCIENTITIC NAME \\
\hline EPA-001-001 & TA-57 & Elk & Cervus canadensis \\
\hline EPA-001-001 & TA-57 & Gopher & Thomomys talpoides \\
\hline EPA-001-001 & TA-57 & Rabbit & Sylvilagus sp. \\
\hline EPA-02A-007 & TA-16 & Coyote & Canis latrans \\
\hline EPA-02A-007 & TA-16 & Elk & Cervus canadensis \\
\hline EPA-02A-007 & TA-16 & Gopher & Thomomys talpoides \\
\hline EPA-02A-007 & TA-16 & Mule deer & Odocoileus hemionus \\
\hline EPA-03A-020 & TA-02 & Aquatic beetle & Colcoptera \\
\hline EPA-03A-020 & TA-02 & Chickadee & Parus sp. \\
\hline EPA-03A-020 & TA-02 & Chipmunk & Eutamias sp. \\
\hline EPA-03A-020 & TA-02 & Coyote & Canis latrans \\
\hline EPA-03A-020 & TA-02 & Decr mouse & Peromyscus maniculatus \\
\hline EPA-03A-020 & TA-02 & Fox & Vulpes vulpes \\
\hline EPA-03A-020 & TA-02 & Mourning cloak butterfly & Nymphalis antiopa \\
\hline EPA-03A-020 & TA-02 & Mule deer & Odocoileus hemionus \\
\hline EPA-03A-020 & TA-02 & Raccoon & Procyon lotor \\
\hline EPA-03A-020 & TA-02 & Vole & Microtus sp. \\
\hline EPA-03A-021 & TA-03 & Aquatic beetle & Coleoptera \\
\hline EPA-03A-021 & TA-03 & Bloodworm & Chironomidae \\
\hline EPA-03A-021 & TA-03 & Elk & Cervus canadensis \\
\hline EPA-03A-021 & TA-03 & Mayfly larva & Ephemeroptera \\
\hline EPA-03A-021 & TA-03 & Mule deer & Odocoileus hemionus \\
\hline EPA-03A-022 & TA-03 & Dragonfly & Anisoptera \\
\hline EPA-03A-022 & TA- 03 & Eastern fence lizard & Sceloporus undulatus \\
\hline EPA-03A-022 & TA-03 & Elk & Cervus canadensis \\
\hline EPA-03A-022 & TA-03 & June beetle & Scarabacidae \\
\hline EPA-03A-022 & TA-03 & Mule deer & Odocoileus hemionus \\
\hline EPA-03A-022 & TA-03 & Squirrel & Spermophilus sp. \\
\hline EPA-03A-023 & TA- 03 & Damselfly adults & Zygoptera \\
\hline EPA-03A-025 & TA- -03 & Bloodworm & Chironomidae \\
\hline EPA-03A-025 & TA-03 & Raccoon & Procyon lotor \\
\hline EPA-03A-025 & TA-03 & Rattlesnake & Crotalinae \\
\hline EPA-03A-025 & TA-03 & Vole & Microtus sp. \\
\hline EPA-03A-027 & TA-03 & Weasel & Mustela sp. \\
\hline EPA-03A-028 & TA-15 & Elk & Cervus canadensis \\
\hline EPA-03A-028 & TA-15 & Mosquito larva & Culicidae \\
\hline EPA-03A-034 & TA-21 & Squirrel & Spermophilus sp. \\
\hline EPA-03A-034 & TA-21 & Turkey vulture & Cathartes aura \\
\hline EPA-03A-036 & TA-21 & Elk & Cervus canadensis \\
\hline EPA-03A-036 & TA-21 & Mule deer & Odocoileus hemionus \\
\hline EPA-03A-036 & TA-21 & Squirrel & Spermophilus sp. \\
\hline EPA-03A-038 & TA-33 & Coyote & Canis latrans \\
\hline EPA-03A-038 & $\mathrm{TA}-33$ & Rabbit & Sylvilagus sp. \\
\hline EPA-03A-040 & $\mathrm{TA}-43$ & Garter snake & Thamnophis elegans \\
\hline EPA-03A-040 & $\mathrm{TA}-43$ & Raccoon & Procyon lotor \\
\hline EPA-03A-042 & TA-46 & Dragonfly & Anisoptera \\
\hline
\end{tabular}




\begin{tabular}{|c|c|c|c|}
\hline EPA-03A-042 & TA-46 & Mayfly larva & Ephemeroptera \\
\hline EPA-03A-042 & TA-46 & Mosquito larva & Culicidae \\
\hline EPA-03A-043 & TA-46 & Mosquito larva & Culicidae \\
\hline EPA-03A-045 & TA-48 & Chipmunk & Eutamias sp. \\
\hline EPA-03A-045 & TA-48 & Dragonfly larva & Anisoptera \\
\hline EPA-03A-045 & $\mathrm{TA}-48$ & Squirrel & Spermophilus sp. \\
\hline EPA-03A-047 & TA-53 & Lizard & Gekkonidac \\
\hline EPA-03A-047 & TA-53 & Mule dcer & Odocoileus hemionus \\
\hline EPA-03A-048 & TA-53 & Squirrel & Spermophilus sp. \\
\hline EPA-03A-060 & TA-16 & Elk & Cervus canadensis \\
\hline EPA-03A-060 & TA-16 & Mulc decr & Odocoileus hemionus \\
\hline EPA-03A-098 & TA-59 & Raccoon & Procyon lotor \\
\hline EPA-03A-113 & TA-53 & Raccoon & Procyon lotor \\
\hline EPA-03A-113 & TA-53 & Weasel & Mustela sp. \\
\hline EPA-03A-113 & TA-53 & Whiptail lizard & Cnemidophorus sp. \\
\hline EPA-03A-114 & TA-53 & Chipmunk & Eutamias sp. \\
\hline EPA-03A-114 & TA-53 & Rabbit & Sylvilagus sp. \\
\hline EPA-03A-114 & TA-53 & Raccoon & Procyon lotor \\
\hline EPA-03A-114 & TA-53 & Robin & Turdus migratorius \\
\hline EPA-03A-124 & TA-46 & Eastern fence lizard & Sceloporus undulatus \\
\hline EPA-03A-130 & TA-11 & Bear & Ursus americanus \\
\hline EPA-03A-130 & TA-11 & Elk & Cervus canadensis \\
\hline EPA-03A-130 & TA-11 & Gopher & Thomomys talpoides \\
\hline EPA-03A-130 & TA-11 & Mule deer & Odocoileus hemionus \\
\hline EPA-03A-130 & TA-11 & Squirrel & Spermophilus sp. \\
\hline EPA-03A-160 & TA-35 & Eastern fence lizard & Sceloporus undulatus \\
\hline EPA-03A-181 & TA-55 & Dark-eyed junco & Junco hyemalis \\
\hline EPA-03A-181 & TA-55 & Elk & Cervus canadensis \\
\hline EPA-03A-181 & TA-55 & Lincoln sparrow & Melospiza lincolnii \\
\hline EPA-03A-181 & TA-55 & Mule deer & Odocoileus hemionus \\
\hline EPA-03A-181 & $\mathrm{TA}-55$ & Raven & Corvus corax \\
\hline EPA-03A-181 & TA-55 & White crowned sparrow & Zonotrichia leucophrys \\
\hline EPA-04A-016 & TA-48 & Damselfly adults & Zygoptera \\
\hline EPA-04A-070 & TA-16 & Elk & Cervus canadensis \\
\hline EPA-04A-070 & TA-16 & Mule deer & Odocoileus hemionus \\
\hline EPA-04A-083 & TA-16 & Mouse & Cricetidae \\
\hline EPA-04A-083 & TA-16 & Mule deer & Odocoileus hemionus \\
\hline EPA-04A-091 & TA-16 & Elk & Cervus canadensis \\
\hline EPA-04A-091 & TA-16 & Mule deer & Odocoileus hemionus \\
\hline EPA-04A-091 & TA-16 & Squirrel & Spermophilus sp. \\
\hline EPA-04A-092 & TA-16 & Elk & Cervus canadensis \\
\hline EPA-04A-092 & TA-16 & Mule deer & Odocoileus hemionus \\
\hline EPA-04A-092 & TA-16 & Squirrel & Spermophilus sp. \\
\hline EPA-04A-093 & TA-15 & Elk & Cervus canadensis \\
\hline EPA-04A-093 & TA-15 & Mule deer & Odocoileus hemionus \\
\hline EPA-04A-093 & TA-15 & Rabbit & Sylvilagus sp. \\
\hline EPA-04A-093 & TA-15 & Squirrel & Spermophilus sp. \\
\hline EPA-04A-093 & TA-15 & Weasel & Mustela sp. \\
\hline EPA-04A-094 & TA-03 & Damselfly adults & Zygoptera \\
\hline EPA-04A-094 & TA-03 & Damselfly larvae & Zygoptera \\
\hline
\end{tabular}




\begin{tabular}{|c|c|c|c|}
\hline EPA-04A-094 & TA-03 & Dragonfly larva & Anisoptera \\
\hline EPA-04A-094 & TA-03 & Whirligig beetle & Gyrinidae \\
\hline EPA-04A-101 & $\mathrm{TA}-40$ & Eastern fence lizard & Sceloporus undulatus \\
\hline EPA- $04 A-102$ & TA-15 & Elk & Cervus canadensis \\
\hline EPA-04A-102 & TA-15 & Gopher & Thomomys talpoides \\
\hline EPA-04A-103 & TA-15 & Elk & Cervus canadensis \\
\hline EPA-04A-109 & TA-03 & Chipmunk & Eutamias sp. \\
\hline EPA-04A-109 & TA-03 & Damselfly adults & Zygoptcra \\
\hline EPA-04A-109 & TA-03 & Elk & Cervus canadensis \\
\hline EPA-04A-109 & TA-03 & Fly larya & Dintera \\
\hline EPA-04A-109 & TA-03 & Isopod & Isopoda \\
\hline EPA-04A-109 & TA-03 & Raccoon & Procyon lotor \\
\hline EPA-04A-109 & TA-03 & Raven & Corvus corcur \\
\hline EPA-04A-109 & TA-03 & Red squirrcl & Sciurus arizonensis \\
\hline EPA-04A-109 & TA-03 & Squirrel & Spermophilus sp. \\
\hline EPA-04A-115 & TA-08 & Damselfly adults & Zygoptera \\
\hline EPA-04A-115 & TA-08 & Elk & Cervus canadensis \\
\hline EPA-04A-115 & TA-08 & Mosquito larva & Culicidae \\
\hline EPA-04A-126 & TA-48 & Dragonfly & Anisoptera \\
\hline EPA-04A-126 & TA-48 & Raccoon & Procyon lotor \\
\hline EPA-04A-127 & TA-35 & Eastern fence lizard & Sceloporus undulatus \\
\hline EPA-04A-127 & TA-35 & Squirrel & Spermophilus sp. \\
\hline EPA-04A-131 & TA-48 & Rabbit & Sylvilagus sp. \\
\hline EPA-04A-131 & TA-48 & Raccoon & Procyon lotor \\
\hline EPA-04A-131 & TA-48 & Squirrel & Spermophilus sp. \\
\hline EPA-04A-137 & TA-48 & Raccoon & Procyon lotor \\
\hline EPA-04A-140 & TA-03 & Rabbit & Sylvilagus sp. \\
\hline EPA-04A-140 & TA-03 & Raccoon & Procyon lotor \\
\hline EPA-04A-140 & TA-03 & Squirrel & Spermophilus sp. \\
\hline EPA-04A-141 & TA-39 & Gopher & Thomomys talpoides \\
\hline EPA-04A-142 & TA-21 & Eastern fence lizard & Sceloporus undulatus \\
\hline EPA-04A-142 & TA-21 & Gopher & Thomomys talpoides \\
\hline EPA-04A-142 & TA-21 & Mule deer & Odocoileus hemionus \\
\hline EPA-04A-143 & TA-15 & Coyote & Canis latrans \\
\hline EPA-04A-143 & TA-15 & Elk & Cervus canadensis \\
\hline EPA-04A-143 & TA-15 & Mule deer & Odocoileus hemionus \\
\hline EPA-04A-147 & TA-33 & Elk & Cervus canadensis \\
\hline EPA-04A-147 & TA-33 & Mule deer & Odocoileus hemionus \\
\hline EPA-04A-147 & TA-33 & Pinyon jay & Gymnorhinus cyanocephalus \\
\hline EPA-04A-147 & TA-33 & Rabbit & Sylvilagus sp. \\
\hline EPA-04A-147 & TA-33 & Raccoon & Procyon lotor \\
\hline EPA-04A-15] & TA-03 & Raccoon & Procyon lotor \\
\hline EPA-04A-152 & TA-48 & Damselfly adults & Zygoptera \\
\hline EPA-04A-152 & TA-48 & Mayfly larva & Ephemeroptera \\
\hline EPA-04A-152 & TA-48 & Raccoon & Procyon lotor \\
\hline EPA-04A-155 & TA-09 & Elk & Cervus canadensis \\
\hline EPA-04A-155 & TA-09 & Mule deer & Odocoileus hemionus \\
\hline EPA-04A-156 & TA-39 & Elk & Cervus canadensis \\
\hline EPA-04A-156 & TA-39 & Gopher & Thomomys talpoides \\
\hline EPA-04A-157 & TA-16 & Coyote & Canis latrans \\
\hline
\end{tabular}




\begin{tabular}{|c|c|c|c|}
\hline EPA-04A-157 & TA-16 & Elk & Cervus canadensis \\
\hline EPA-04A-157 & TA-16 & Gopher & Thomomys talpoides \\
\hline EPA-04A-157 & TA-16 & Mule deer & Odocoileus hemionus \\
\hline EPA-04A-157 & TA-16 & Squirrel & Spermophilus $\mathrm{sp}$. \\
\hline EPA-04A-157 & TA-16 & Water striders & Gerridae \\
\hline EPA-04A-157 & TA-16 & Whirligig beetle & Gyrinidae \\
\hline EPA-04A-165 & $\mathrm{TA}-0$ & Mule deer & Odocoilens hemionus \\
\hline EPA-04A-176 & TA-0 & Gopher & Thomomys talpoides \\
\hline EPA- $04 A-182$ & TA-21 & Eastern fence lizard & Sceloporus undulatus \\
\hline EPA-04A-182 & TA-21 & Gopher & Thomomys talpoides \\
\hline EPA-04A-182 & TA-21 & Squirrel & Spermophilus sp. \\
\hline EPA-05A-052 & TA-16 & Eastern fence lizard & Sceloporus undulatus \\
\hline EPA-05A-052 & TA-16 & Elk & Cervus canadensis \\
\hline EPA-05A-052 & TA-16 & Gopher & Thomomys talpoides \\
\hline EPA-05A-052 & TA-16 & Mule deer & Odocoilens hemionus \\
\hline EPA-05A-053 & TA-16 & Eastern fence lizard & Sceloporus undulatus \\
\hline EPA-05A-053 & TA-16 & Mule deer & Odocoileus hemionus \\
\hline EPA-05A-053 & TA-16 & Porcupine & Erethizon dorsatum \\
\hline EPA-05A-053 & TA-16 & Short horned lizard & Phrynosoma douglassi \\
\hline EPA-05A-054 & TA-16 & Abert's squirrel & Sciurus aberti \\
\hline EPA-05A-054 & TA-16 & Damselfly larvae & Zygoptera \\
\hline EPA-05A-054 & TA-16 & Elk & Cervus canadensis \\
\hline EPA-05A-054 & TA-16 & Fly larva & Diptera \\
\hline EPA-05A-054 & TA-16 & Mayfly larva & Ephemeroptera \\
\hline EPA-05A-054 & TA-16 & Mule deer & Odocoileus hemionus \\
\hline EPA-05A-054 & TA-16 & Porcupine & Erethizon dorsatum \\
\hline EPA-05A-054 & TA-16 & Rabbit & Sylvilagus sp. \\
\hline EPA-05A-054 & TA-16 & Squirrel & Spermophilus sp. \\
\hline EPA-05A-055 & TA-16 & Eastern fence lizard & Sceloporus undulatus \\
\hline EPA-05A-055 & TA-16 & Squirrel & Spermophilus sp. \\
\hline EPA-05A-056 & TA-16 & Eastern fence lizard & Sceloporus undulatus \\
\hline EPA-05A-056 & TA-16 & Elk & Cervus canadensis \\
\hline EPA-05A-056 & TA-16 & Mule deer & Odocoileus hemionus \\
\hline EPA-05A-056 & TA-16 & Squirrel & Spermophilus sp. \\
\hline EPA-05A-057 & TA-16 & Elk & Cervus canadensis \\
\hline EPA-05A-057 & TA-16 & Mule deer & Odocoileus hemionus \\
\hline EPA-05A-058 & TA-16 & Dragonfly & Anisoptera \\
\hline EPA-05A-058 & TA-16 & Elk & Cervus canadensis \\
\hline EPA-05A-058 & TA-15 & Mule deer & Odocoileus hemionus \\
\hline EPA-05A-058 & TA-16 & Rabbit & Sylvilagus sp. \\
\hline EPA-05A-058 & TA-16 & Squirrel & Spermophilus sp. \\
\hline EPA-05A-058 & TA-15 & Water striders & Gerridae \\
\hline EPA-05A-061 & TA-16 & Elk & Cervus canadensis \\
\hline EPA-05A-061 & TA-16 & Mule deer & Odocoileus hemionus \\
\hline EPA-05A-062 & TA-16 & Eastern fence lizard & Sceloporus undulatus \\
\hline EPA-05A-062 & TA-16 & Mule deer & Odocoileus hemionus \\
\hline EPA-05A-062 & TA-16 & Rabbit & Sylvilagus sp. \\
\hline EPA-05A-062 & TA-16 & Squirrel & Spermophilus sp. \\
\hline EPA-05A-063 & TA-16 & Chipmunk & Eutamias sp. \\
\hline EPA-05A-063 & TA-16 & Elk & Cervus canadensis \\
\hline
\end{tabular}




\begin{tabular}{|c|c|c|c|}
\hline EPA-05A-063 & TA-16 & Mule deer & Odocoileus hemionus \\
\hline EPA-05A-063 & TA-16 & Raccoon & Procyon lotor \\
\hline EPA-05A-063 & TA-16 & Skunk & Mephitis sp. \\
\hline EPA-05A-063 & TA-16 & Squirrel & Spermophilus sp. \\
\hline EPA-05A-066 & TA-09 & Elk & Cervus canadensis \\
\hline EPA-05A-066 & TA-09 & Mule deer & Odocoileus hemionus \\
\hline EPA-05A-066 & TA-09 & Squirrel & Spermophilus sp. \\
\hline EPA-05A-067 & TA-09 & Coyote & Canis latrans \\
\hline EPA-05A-067 & TA-09 & Elk & Cervus canadensis \\
\hline EPA-05A-068 & TA-09 & Eastern fence lizard & Sceloporus undulatus \\
\hline EPA-05A-068 & TA-09 & Elk & Cervus canadensis \\
\hline EPA-05A-069 & TA-11 & Bear & Ursus americanus \\
\hline EPA-05A-069 & TA-11 & Elk & Cervus canadensis \\
\hline EPA-05A-069 & TA-11 & Mule deer & Odocoileus hemionus \\
\hline EPA-05A-069 & TA-11 & Water striders & Gerridae \\
\hline EPA-05A-071 & TA-16 & Eastern fence lizard & Sceloporus undulatus \\
\hline EPA-05A-071 & TA-16 & Elk & Cervus canadensis \\
\hline EPA-05A-071 & TA-16 & Mule deer & Odocoileus hemionus \\
\hline EPA-05A-071 & TA-16 & Shrew & Sorex sp. \\
\hline EPA-05A-072 & TA-16 & Elk & Cervus canadensis \\
\hline EPA-05A-072 & TA-16 & Mule deer & Odocoileus hemionus \\
\hline EPA-05A-096 & TA-11 & Bear & Ursus americanus \\
\hline EPA-05A-096 & TA-11 & Coyote & Canis latrans \\
\hline EPA-05A-096 & TA-11 & Elk & Cervus canadensis \\
\hline EPA-05A-096 & TA-11 & Mule deer & Odocoileus hemionus \\
\hline EPA-05A-096 & TA-11 & Squirrel & Spermophilus sp. \\
\hline EPA-05A-097 & TA-11 & Mule deer & Odocoileus hemionus \\
\hline EPA-05A-149 & TA-16 & Elk & Cervus canadensis \\
\hline EPA-05A-149 & TA-16 & Mule deer & Odocoileus hemionus \\
\hline EPA-05A-159 & TA-16 & Elk & Cervus canadensis \\
\hline EPA-05A-159 & TA-16 & Mule deer & Odocoileus hemionus \\
\hline EPA-06A-073 & TA-16 & Chorus frog & Pseudacris triseriata \\
\hline EPA-06A-073 & TA-16 & Mule deer & Odocoileus hemionus \\
\hline EPA-06A-073 & TA-16 & Water striders & Gerridae \\
\hline EPA-06A-073 & TA-16 & Whirligig beetle & Gyrinidae \\
\hline EPA-06A-074 & TA-08 & Damselfly adults & Zygoptera \\
\hline EPA-06A-074 & TA-08 & Elk & Cervus canadensis \\
\hline EPA-06A-074 & TA-08 & Mosquito larva & Culicidae \\
\hline EPA-06A-075 & TA-08 & Aquatic beetle & Coleoptera \\
\hline EPA-06A-075 & TA-08 & Coyote & Canis latrans \\
\hline EPA-06A-075 & TA-08 & Elk & Cervis canadensis \\
\hline EPA-06A-075 & TA-08 & Mosquito larva & Culicidae \\
\hline EPA-06A-075 & TA-08 & Mule deer & Odocoileus hemionus \\
\hline EPA-06A-075 & TA-08 & Squirrel & Spermophilus sp. \\
\hline EPA-06A-079 & TA -40 & Eastern fence lizard & Sceloporus undulatus \\
\hline EPA-06A-079 & TA-40 & Elk & Cervus canadensis \\
\hline EPA-06A-080 & TA-40 & Bear & Ursus americanus \\
\hline EPA-06A-080 & TA-40 & Eastem fence lizard & Sceloporus undulatus \\
\hline EPA-06A-080 & TA-40 & Elk & Cervus canadensis \\
\hline EPA-06A-080 & TA -40 & Mule deer & Odocoileus hemionus \\
\hline
\end{tabular}




\begin{tabular}{|c|c|c|c|}
\hline EPA-06A-099 & $\mathrm{TA}-40$ & Elk & Cervus canadensis \\
\hline EPA-06A-106 & TA-36 & Woodrat & Neotoma sp. \\
\hline EPA-06A-123 & TA-15 & Elk & Cervus canadensis \\
\hline EPA-06A-123 & TA-15 & Mule deer & Odocoileus hemionus \\
\hline EPA-06A-132 & TA-35 & Squirrel & Spermophilus sp. \\
\hline EPA-128-128 & TA-22 & Abert's squirrel & Sciurus aberti \\
\hline EPA-128-128 & TA-22 & Aquatic beetle & Coleoptera \\
\hline EPA-128-128 & TA-22 & Chickadee & Partus sp. \\
\hline EPA-128-128 & TA-22 & Bohcat & Felis rufus \\
\hline EPA-128-128 & TA-22 & Coyote & Canis latrans \\
\hline EPA-128-128 & TA-22 & Decr mouse & Peromyscus maniculatus \\
\hline EPA-128-128 & TA-22 & Earthworm & Annelida \\
\hline EPA-128-128 & TA-22 & Elk & Cervus canadensis \\
\hline EPA-128-128 & TA-22 & Great horned owl & Bubo virginianus \\
\hline EPA-128-128 & TA-22 & Mule deer & Odocoileus hemionus \\
\hline EPA-128-128 & TA-22 & Raccoon & Procyon lotor \\
\hline EPA-128-128 & TA-22 & Raven & Corvus corax \\
\hline EPA-128-128 & TA-22 & Shrimp & Amphipoda \\
\hline EPA-128-128 & TA-22 & Water striders & Gerridae \\
\hline EPA-SSS-01S & TA-03 & Coyote & Canis latranss \\
\hline EPA-SSS-01S & TA-03 & Elk & Cervus canadensis \\
\hline EPA-SSS-01S & TA-03 & Raccoon & Procyon lotor \\
\hline EPA-SSS-02S & TA-09 & Coyote & Canis latrans \\
\hline EPA-SSS-02S & TA-09 & Elk & Cervus canadensis \\
\hline EPA-SSS-03S & TA-16 & Damselfly adults & Zygoptera \\
\hline EPA-SSS-03S & TA-16 & Elk & Cervus canadensis \\
\hline EPA-SSS-03S & TA-16 & Mule deer & Odocoileus hemionus \\
\hline EPA-SSS-03S & TA-16 & Squirrel & Spermophilus sp \\
\hline EPA-SSS-03S & TA-16 & Water striders & Gerridae \\
\hline EPA-SSS-04S & TA-18 & Damselfly adults & Zygoptera \\
\hline EPA-SSS-04S & TA-18 & Dragonfly & Anisoptera \\
\hline EPA-SSS-04S & TA-18 & Elk & Cervus canadensis \\
\hline EPA-SSS-04S & TA-18 & Fly larva & Diptera \\
\hline EPA-SSS-04S & TA-18 & Gopher & Thomomys talpoides \\
\hline EPA-SSS-04S & TA-18 & Mayfly larva & Ephemeroptera \\
\hline EPA-SSS-04S & TA-18 & Mule deer & Odocoileus hemionus \\
\hline EPA-SSS-04S & TA-18 & Raccoon & Procyon lotor \\
\hline EPA-SSS-04S & TA-18 & Snail & Gastropoda \\
\hline EPA-SSS-04S & TA-18 & Water striders & Gerridae \\
\hline EPA-SSS-05S & TA-21 & Mule deer & Odocoileus hemionus \\
\hline EPA-SSS-05S & TA-21 & Squirrel & Spermophilus sp. \\
\hline EPA-SSS-09S & TA-53 & Turkey vulture & Cathartes aura \\
\hline EPA-SSS-10S & TA-35 & Elk & Cervus canadensis \\
\hline EPA-SSS-10S & TA-35 & Mule deer & Odocoileus hemionus \\
\hline
\end{tabular}


Table 11A. Outfalls with Hydrophytic Vegetation

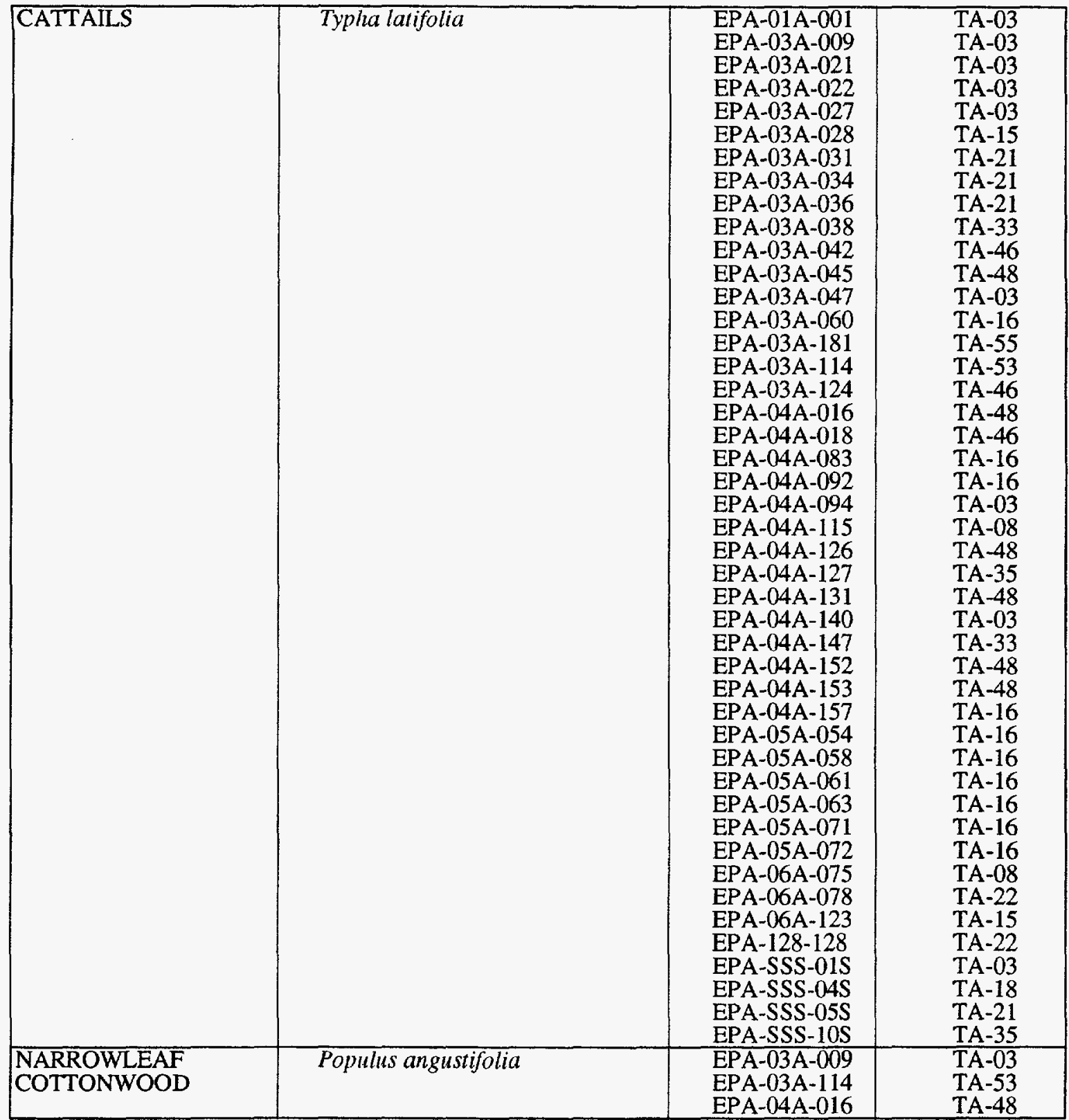




\begin{tabular}{|c|c|c|c|}
\hline SEDGES/RUSHES & Carex sp/Juncus sp. & $\begin{array}{l}\text { EPA-03A-022 } \\
\text { EPA-03A-036 } \\
\text { EPA-03A-045 } \\
\text { EPA-03A-047 } \\
\text { EPA-03A-060 } \\
\text { EPA-03A-114 } \\
\text { EPA-04A-094 } \\
\text { EPA-04A-115 } \\
\text { EPA-04A-126 } \\
\text { EPA-04A-140 } \\
\text { EPA-04A-153 } \\
\text { EPA-04A-157 } \\
\text { EPA-05A-054 } \\
\text { EPA-05A-061 } \\
\text { EPA-05A-066 } \\
\text { EPA-05A-071 } \\
\text { EPA-051-051 } \\
\text { EPA-128-128 }\end{array}$ & $\begin{array}{l}\text { TA-03 } \\
\text { TA-21 } \\
\text { TA-48 } \\
\text { TA-53 } \\
\text { TA-16 } \\
\text { TA-53 } \\
\text { TA-03 } \\
\text { TA-08 } \\
\text { TA-48 } \\
\text { TA-03 } \\
\text { TA-48 } \\
\text { TA-16 } \\
\text { TA-16 } \\
\text { TA-16 } \\
\text { TA-09 } \\
\text { TA-16 } \\
\text { TA-50 } \\
\text { TA-22 }\end{array}$ \\
\hline TAMARISK & Tamarix pentandra & EPA-03A-034 & TA-21 \\
\hline WATERCRESS & Rorippa sp. & EPA-04A-094 & TA-03 \\
\hline WILLOW & Salix sp. & $\begin{array}{l}\text { EPA-03A-009 } \\
\text { EPA-03A-022 } \\
\text { EPA-03A-036 } \\
\text { EPA-03A-040 } \\
\text { EPA-03A-098 } \\
\text { EPA-03A-114 } \\
\text { EPA-03A-181 } \\
\text { EPA-04A-016 } \\
\text { EPA-04A-091 } \\
\text { EPA-04A-092 } \\
\text { EPA-04A-093 } \\
\text { EPA-04A-115 } \\
\text { EPA-04A-126 } \\
\text { EPA-04A-127 } \\
\text { EPA-04A-147 } \\
\text { EPA-04A-152 } \\
\text { EPA-05A-053 } \\
\text { EPA-05A-071 } \\
\text { EPA-06A-132 } \\
\text { EPA-SSS-04S }\end{array}$ & $\begin{array}{l}\text { TA-03 } \\
\text { TA-03 } \\
\text { TA-21 } \\
\text { TA-43 } \\
\text { TA-59 } \\
\text { TA-53 } \\
\text { TA-55 } \\
\text { TA-48 } \\
\text { TA-16 } \\
\text { TA-16 } \\
\text { TA-15 } \\
\text { TA-08 } \\
\text { TA-48 } \\
\text { TA-35 } \\
\text { TA-33 } \\
\text { TA-48 } \\
\text { TA-16 } \\
\text { TA-16 } \\
\text { TA-35 } \\
\text { TA-18 }\end{array}$ \\
\hline BARNYARD GRASS & Echinochloa crusgalli & EPA-06A-073 & TA-16 \\
\hline
\end{tabular}


Table 11B. Outfalls with Hydrophytic Vegetation

\begin{tabular}{|c|c|c|c|}
\hline OUTFALL NO. & TA & COMMON NAME & SCIENTIFIC NAME \\
\hline EPA-01A-001 & TA-03 & Cattails & Typha latifolia \\
\hline EPA-03A-009 & TA-03 & Cattails & Typha latifolia \\
\hline EPA-03A-009 & TA-03 & Narrowleaf cottonwood & Populus angustifolia \\
\hline EPA-03A-009 & TA-03 & Willow & Salix sp. \\
\hline EPA-03A-021 & TA-03 & Cattails & Typha latifolia \\
\hline EPA-03A-022 & TA-03 & Cattails & Typha latifolia \\
\hline EPA-03A-022 & TA-03 & Sedges/Rushes & Carex sp./Juncus sp. \\
\hline EPA-03A-022 & TA-03 & Willow & Salix sp. \\
\hline EPA-03A-027 & TA-03 & Cattails & Typha latifolia \\
\hline EPA-03A-028 & TA-15 & Cattails & Typha latifolia \\
\hline EPA-03A-031 & TA-21 & Cattails & Typha latifolia \\
\hline EPA-03A-034 & TA-21 & Cattails & Typha latifolia \\
\hline EPA-03A-034 & TA-21 & Tamarisk & Tamarix pentandra \\
\hline EPA-03A-036 & TA-21 & Cattails & Typha latifolia \\
\hline EPA-03A-036 & TA-21 & Sedges/Rushes & Carex sp./Juncus sp. \\
\hline EPA-03A-036 & TA-21 & Willow & Salix sp. \\
\hline EPA-03A-038 & TA-33 & Cattails & Typha latifolia \\
\hline EPA-03A-040 & TA-43 & Willow & Salix sp. \\
\hline EPA-03A-042 & TA-46 & Cattails & Typha latifolia \\
\hline EPA-03A-045 & TA-48 & Cattails & Typha latifolia \\
\hline EPA-03A-045 & TA-48 & Sedges/Rushes & Carex sp./Juncus sp. \\
\hline EPA-03A-047 & TA-03 & Cattails & Typha latifolia \\
\hline EPA-03A-047 & TA-53 & Sedges/Rushes & Carex sp./Juncus sp. \\
\hline EPA-03A-060 & TA-16 & Cattails & Typha latifolia \\
\hline EPA-03A-060 & TA-16 & Sedges/Rushes & Carex sp./Juncus sp. \\
\hline EPA-03A-098 & TA-59 & Willow & Salix sp. \\
\hline EPA-03A-114 & TA-53 & Cattails & Typha latifolia \\
\hline EPA-03A-114 & TA-53 & Narrowleaf cottonwood & Populus angustifolia \\
\hline EPA-03A-114 & TA-53 & Sedges/Rushes & Carex sp./Juncus sp. \\
\hline EPA-03A-114 & TA-53 & Willow & Salix sp. \\
\hline EPA-03A-124 & TA-46 & Cattails & Typha latifolia \\
\hline EPA-03A-181 & TA-55 & Cattails & Typha latifolia \\
\hline EPA-03A-181 & TA-55 & Willow & Salix sp. \\
\hline EPA-04A-016 & TA-48 & Cattails & Typha latifolia \\
\hline EPA-04A-016 & TA-48 & Narrowleaf cottonwood & Populus angustifolia \\
\hline EPA-04A-016 & TA-48 & Willow & Salix sp. \\
\hline EPA-04A-018 & TA-46 & Cattails & Typha latifolia \\
\hline EPA-04A-083 & TA-16 & Cattails & Typha latifolia \\
\hline EPA-04A-091 & TA-16 & Willow & Salix sp. \\
\hline EPA-04A-092 & TA-16 & Cattails & Typha latifolia \\
\hline EPA-04A-092 & TA-16 & Willow & Salix sp. \\
\hline EPA-04A-093 & TA-15 & Willow & Salix sp. \\
\hline EPA-04A-094 & TA-03 & Cattails & Typha latifolia \\
\hline EPA-04A-094 & TA-03 & Sedges/Rushes & Carex sp./Juncus sp. \\
\hline EPA-04A-094 & TA-03 & Watercress & Rorippa sp. \\
\hline EPA-04A-115 & TA-08 & Cattails & Typha latifolia \\
\hline EPA-04A-115 & TA- 08 & Sedges/Rushes & Carex sp./Juncus sp. \\
\hline
\end{tabular}




\begin{tabular}{|c|c|c|c|}
\hline OUTFALL NO. & $\overline{\mathbf{T A}}$ & COMMON NAME & SCEENTIFIC NAME \\
\hline EPA-04A-115 & TA-08 & Willow & Salix sp. \\
\hline EPA-04A-126 & TA-48 & Cattails & Typha latifolia \\
\hline EPA-04A-126 & TA-48 & Sedges/Rushes & Carex sp./Juncus sp. \\
\hline EPA-04A-126 & TA-48 & Willow & Salix sp. \\
\hline EPA-04A-127 & TA-35 & Cattails & Typha latifolia \\
\hline EPA-04A-127 & TA-35 & Willow & Salix sp. \\
\hline EPA-04A-131 & TA-48 & Cattails & Typha latifolia \\
\hline EPA-04A-140 & TA-03 & Cattails & Typha latifolia \\
\hline EPA-04A-140 & TA-03 & Sedges/Rushes & Carex sp./Juncus sp. \\
\hline EPA-04A-147 & TA-33 & Cattails & Typha latifolia \\
\hline EPA-04A-147 & TA-33 & Willow & Salix sp. \\
\hline EPA-04A-152 & TA-48 & Cattails & Typha latifolia \\
\hline EPA-04A-152 & TA-48 & Willow & Salix sp. \\
\hline EPA-04A-153 & TA-48 & Cattails & Typha latifolia \\
\hline EPA-04A-153 & TA-48 & Sedges/Rushes & Carex sp./Juncus sp. \\
\hline EPA-04A-157 & TA-16 & Cattails & Typha latifolia \\
\hline EPA-04A-157 & TA-16 & Sedges/Rushes & Carex sp./Juncus sp. \\
\hline EPA-051-051 & TA-50 & Sedges/Rushes & Carex sp./Juncus sp. \\
\hline EPA-05A-053 & TA-16 & Willow & Salix sp. \\
\hline EPA-05A-054 & TA-16 & Cattails & Typha latifolia \\
\hline EPA-05A-054 & TA-16 & Sedges/Rushes & Carex sp./Juncus sp. \\
\hline EPA-05A-058 & TA-16 & Cattails & Typha latifolia \\
\hline EPA-05A-061 & TA-16 & Cattails & Typha latifolia \\
\hline EPA-05A-061 & TA-16 & Sedges/Rushes & Carex sp./Juncus sp. \\
\hline EPA-05A-063 & TA-16 & Cattails & Typha latifolia \\
\hline EPA-05A-066 & TA-09 & Sedges/Rushes & Carex sp./Juncus sp. \\
\hline EPA-05A-071 & TA-16 & Cattails & Typha latifolia \\
\hline EPA-05A-071 & TA-16 & Sedges/Rushes & Carex sp./Juncus sp. \\
\hline EPA-05A-071 & TA-16 & Willow & Salix sp. \\
\hline EPA-05A-072 & TA-16 & Cattails & Typha latifolia \\
\hline EPA-06A-073 & TA-16 & Barnyard grass & Echinochioa crusgalli \\
\hline EPA-06A-075 & TA-08 & Cattails & Typha latifolia \\
\hline EPA-06A-078 & TA-22 & Cattails & Typha latifolia \\
\hline EPA-06A-123 & TA-15 & Cattails & Typha latifolia \\
\hline EPA-06A-132 & TA-35 & Willow & Salix sp. \\
\hline EPA-128-128 & TA-22 & Cattails & Typha latifolia \\
\hline EPA-128-128 & TA-22 & Sedges/Rushes & Carex sp./Juncus sp. \\
\hline EPA-SSS-01S & TA-03 & Cattails & Typha latifolia \\
\hline EPA-SSS-04S & TA-18 & Cattails & Typha latifolia \\
\hline EPA-SSS-04S & TA-18 & Willow & Salix sp. \\
\hline EPA-SSS-05S & TA-21 & Cattails & Typha latifolia \\
\hline EPA-SSS-10S & TA-35 & Cattails & Typha latifolia \\
\hline
\end{tabular}


TABLE 12. Miscellaneous Observations

\begin{tabular}{|c|c|c|}
\hline OUTFALL & TA & COMMENTS \\
\hline $03 \mathrm{~A}-022$ & 03 & Tarp in channel \\
\hline $03 \mathrm{~A}-023$ & 03 & $\begin{array}{l}\text { No sign, bundles absorbent } \\
\text { boom }\end{array}$ \\
\hline $03 \mathrm{~A}-040$ & 43 & Stryofoaun beads in channel \\
\hline $03 \mathrm{~A}-045$ & 48 & Erosion \\
\hline $03 \mathrm{~A}-148$ & 03 & No sign \\
\hline $03 \mathrm{~A}-181$ & 55 & Erosion \\
\hline $04 \mathrm{~A}-083$ & 16 & $\begin{array}{l}\text { Small white particles in } \\
\text { channel }\end{array}$ \\
\hline 04A-092 & 16 & $\begin{array}{l}\text { Number on sign different } \\
\text { number in print out }(04 \mathrm{~A})\end{array}$ \\
\hline $04 \mathrm{~A}-094$ & 03 & Dumping, debris in channel \\
\hline $04 \mathrm{~A}-109$ & 03 & Asphalt in channel \\
\hline $04 A-143$ & 15 & Oil sprays reaching channel \\
\hline $04 A-151$ & 03 & Gray sediment lining channe \\
\hline $06 \mathrm{~A}-099$ & 40 & Grate covering pipe open \\
\hline $06 A-100$ & 40 & Erosion \\
\hline $06 \mathrm{~A}-132$ & 35 & $\begin{array}{l}\text { Debris in channel, discarded } \\
\text { pipe }\end{array}$ \\
\hline
\end{tabular}


Table 13. Surface Water Flow at Surveyed Outfalls

\begin{tabular}{|c|c|c|}
\hline NPDES Number & TA & Length of flow \\
\hline EPA-128-128 & 22 & At least $1380 \mathrm{ft}$ \\
\hline EPA-051-051 & 50 & N/A \\
\hline EPA-001-001 & 57 & N/A \\
\hline EPA-01A-001 & 3 & $\begin{array}{l}\text { Flow from combined outfalls approximately } \\
\qquad 2 \text { miles }\end{array}$ \\
\hline EPA-02A-007 & 16 & $950 \mathrm{ft}$. \\
\hline EPA-02A-129 & 21 & $\mathrm{~N} / \mathrm{A}$ \\
\hline EPA-03A-009 & 3 & Undetermined \\
\hline EPA-03A-020 & 2 & Undetermined \\
\hline EPA-03A-021 & 3 & $200 \mathrm{ft}$. \\
\hline EPA-03A-022 & 3 & $350-400 \mathrm{ft}$. \\
\hline EPA-03A-023 & 3 & $330 \mathrm{ft}$. \\
\hline EPA-03A-024 & 3 & N/A \\
\hline EPA-03A-025 & 3 & $30 \mathrm{ft}$. \\
\hline EPA-03A-027 & 3 & $\begin{array}{l}\text { Flow from combined outfalls approximately } \\
2 \text { miles } \\
\end{array}$ \\
\hline EPA-03A-028 & 15 & $6 \mathrm{ft}$. \\
\hline EPA-03A-031 & 21 & Undetermined \\
\hline EPA-03A-032 & 21 & $2 \mathrm{ft}$. \\
\hline EPA-03A-034 & 21 & $30 \mathrm{ft}$. \\
\hline EPA-03A-035 & 21 & N/A \\
\hline EPA-03A-036 & 21 & N/A \\
\hline EPA-03A-037 & 21 & N/A \\
\hline EPA-03A-038 & 33 & $30 \mathrm{ft}$. \\
\hline EPA-03A-040 & 43 & $250-300 \mathrm{ft}$. \\
\hline EPA-03A-042 & 46 & Intermittent for $125 \mathrm{ft}$. \\
\hline EPA-03A-043 & 46 & Undetermined \\
\hline EPA-03A-045 & 48 & Intermittent for $250 \mathrm{ft}$. \\
\hline EPA-03A-047 & 53 & Intermittent for $435 \mathrm{ft}$. \\
\hline EPA-03A-048 & 53 & Undetermined \\
\hline EPA-03A-049 & 53 & Undetermined \\
\hline EPA-03A-060 & 16 & $20 \mathrm{ft}$. \\
\hline EPA-03A-098 & 59 & $110 \mathrm{ft}$. \\
\hline EPA-03A-113 & 53 & $500-800 \mathrm{ft}$. \\
\hline EPA-03A-114 & 53 & $125 \mathrm{ft}$. \\
\hline EPA-03A-124 & 46 & $20 \mathrm{ft}$. \\
\hline EPA-03A-125 & 53 & N/A \\
\hline EPA-03A-130 & 11 & N/A \\
\hline EPA-03A-136 & 46 & $\mathrm{~N} / \mathrm{A}$ \\
\hline EPA-03A-145 & 53 & $1.5 \mathrm{ft}$. \\
\hline EPA-03A-146 & 53 & $\mathrm{~N} / \mathrm{A}$ \\
\hline EPA-03A-148 & 3 & At least $45 \mathrm{ft}$. \\
\hline EPA-03A-158 & 21 & Undetermined \\
\hline EPA-03A-160 & 35 & $\mathrm{~N} / \mathrm{A}$ \\
\hline EPA-03A-181 & 55 & $390 \mathrm{ft}$. \\
\hline EPA-04A-013 & 46 & N/A \\
\hline EPA-04A-014 & 46 & Undetermined \\
\hline EPA-04A-016 & 48 & Intermittent for $150 \mathrm{ft}$. \\
\hline
\end{tabular}




\begin{tabular}{|c|c|c|}
\hline NPDES Number & TA & Length of flow \\
\hline EPA-04A-018 & 46 & N/A \\
\hline EPA-04A-070 & 16 & $40 \mathrm{ft}$ \\
\hline EPA-04A-083 & 16 & $295 \mathrm{ft}$. \\
\hline EPA-04A-091 & 16 & $\mathrm{~N} / \mathrm{A}$ \\
\hline EPA-04A-092 & 16 & $2 \mathrm{ft}$. \\
\hline EPA-04A-093 & 15 & $60 \mathrm{ft}$. \\
\hline EPA-04A-094 & 3 & Undetermined \\
\hline EPA-04A-101 & 40 & Immediately disappears \\
\hline EPA-04A-102 & 15 & N/A \\
\hline EPA-04A-103 & 15 & $\mathrm{~N} / \mathrm{A}$ \\
\hline EPA-04A-109 & 3 & $\begin{array}{l}\text { Flow from combined outfalls approximately } \\
\qquad 2 \text { miles }\end{array}$ \\
\hline EPA-04A-115 & 8 & $\mathrm{~N} / \mathrm{A}$ \\
\hline EPA-04A-117 & 46 & N/A \\
\hline EPA-04A-118 & 0 & Not surveycd \\
\hline EPA-04A-126 & 48 & Approximately $500 \mathrm{ft}$. \\
\hline EPA-04A-127 & 35 & Intermittent for $60 \mathrm{ft}$. \\
\hline EPA-04A-131 & 48 & $45 \mathrm{ft}$. \\
\hline EPA-04A-133 & 53 & Not surveyed \\
\hline EPA-04A-135 & 53 & N/A \\
\hline EPA-04A-137 & 48 & N/A \\
\hline EPA-04A-139 & 15 & N/A \\
\hline EPA-04A-140 & 3 & $\mathrm{~N} / \mathrm{A}$ \\
\hline EPA-04A-141 & 39 & $30 \mathrm{ft}$. maximum \\
\hline EPA-04A-142 & 21 & $15 \mathrm{ft}$. \\
\hline EPA-04A-143 & 15 & Undetermined \\
\hline EPA-04A-147 & 33 & N/A \\
\hline EPA-04A-151 & 3 & $\begin{array}{l}\text { Flow from combined outfalls approximately } \\
\qquad 2 \text { miles }\end{array}$ \\
\hline EPA-04A-152 & 48 & At least $175 \mathrm{ft}$. \\
\hline EPA-04A-153 & 48 & Intermittent for $160 \mathrm{ft}$. \\
\hline EPA-04A-155 & 9 & N/A \\
\hline EPA-04A-156 & 39 & $\mathrm{~N} / \mathrm{A}$ \\
\hline EPA-04A-157 & 16 & $3000 \mathrm{ft}$. \\
\hline EPA-04A-161 & 0 & Not surveyed \\
\hline EPA-04A-163 & 0 & N/A \\
\hline EPA-04A-164 & 0 & $50 \mathrm{ft}$. \\
\hline EPA-04A-165 & 0 & N/A \\
\hline EPA-04A-166 & 0 & Not surveyed \\
\hline EPA-04A-167 & 0 & Not surveyed \\
\hline EPA-04A-168 & 0 & N/A \\
\hline EPA-04A-169 & 0 & N/A \\
\hline EPA-04A-170 & 0 & $\mathrm{~N} / \mathrm{A}$ \\
\hline EPA-04A-171 & 0 & N/A \\
\hline EPA-04A-172 & 0 & $\mathrm{~N} / \mathrm{A}$ \\
\hline EPA-04A-173 & 0 & N/A \\
\hline EPA-04A-174 & 0 & N/A \\
\hline EPA-04A-175 & 0 & N/A \\
\hline EPA-04A-176 & 0 & N/A \\
\hline
\end{tabular}




\begin{tabular}{|c|c|c|}
\hline NPDES Number & $\mathbf{T A}$ & Length of flow \\
\hline EPA-04A-177 & 0 & Not surveyed \\
\hline EPA-04A-178 & 0 & N/A \\
\hline EPA-04A-179 & 0 & Not surveyed \\
\hline EPA-04A-182 & 21 & $\mathrm{~N} / \mathrm{A}$ \\
\hline EPA-05A-052 & 16 & N/A \\
\hline EPA-05A-053 & 16 & $12 \mathrm{ft}$. \\
\hline EPA-05A-054 & 16 & $1000 \mathrm{ft}$. \\
\hline EPA-05A-055 & 16 & $125 \mathrm{ft}$. \\
\hline EPA-05A-056 & 16 & $400 \mathrm{ft}$. \\
\hline EPA-05A-057 & 16 & N/A \\
\hline EPA-05A-058 & 16 & At least $480 \mathrm{ft}$. \\
\hline EPA-05A-061 & 16 & N/A \\
\hline EPA-05A-062 & 16 & N/A \\
\hline EPA-05A-063 & 16 & N/A \\
\hline EPA-05A-066 & 9 & $90 \mathrm{ft}$. \\
\hline EPA-05A-067 & 9 & N/A \\
\hline EPA-05A-068 & 9 & N/A \\
\hline EPA-05A-069 & 11 & N/A \\
\hline EPA-05A-071 & 16 & N/A \\
\hline EPA-05A-072 & 16 & N/A \\
\hline EPA-05A-096 & 11 & N/A \\
\hline EPA-05A-097 & 11 & N/A \\
\hline EPA-05A-149 & 16 & $\mathrm{~N} / \mathrm{A}$ \\
\hline EPA-05A-154 & 40 & $\mathrm{~N} / \mathrm{A}$ \\
\hline EPA-05A-159 & 16 & N/A \\
\hline EPA-06A-073 & 16 & $280 \mathrm{ft}$. \\
\hline EPA-06A-074 & 8 & $715 \mathrm{ft}$. \\
\hline EPA-06A-075 & 8 & $350 \mathrm{ft}$. \\
\hline EPA-06A-078 & 22 & $146 \mathrm{ft}$ \\
\hline EPA-06A-079 & 40 & N/A \\
\hline EPA-06A-080 & 40 & $1 \mathrm{ft}$. \\
\hline EPA-06A-081 & 40 & N/A \\
\hline EPA-06A-082 & 40 & Immediately disappears \\
\hline EPA-06A-099 & 40 & N/A \\
\hline EPA-06A-100 & 40 & N/A \\
\hline EPA-06A-106 & 36 & $50 \mathrm{ft}$. \\
\hline EPA-06A-123 & 15 & N/A \\
\hline EPA-06A-132 & 35 & $50 \mathrm{ft}$. \\
\hline EPA-SSS-01S & 3 & $\begin{array}{l}\text { Flow from combined outfalls approximately } \\
\qquad 2 \text { miles }\end{array}$ \\
\hline EPA-SSS-02S & 9 & Undetermined \\
\hline EPA-SSS-03S & 16 & Undetermined \\
\hline EPA-SSS-04S & 18 & N/A \\
\hline EPA-SSS-05S & 21 & $300-400 \mathrm{ft}$. \\
\hline EPA-SSS-06S & 41 & N/A \\
\hline EPA-SSS-07S & 46 & $\mathrm{~N} / \mathrm{A}$ \\
\hline EPA-SSS-09S & 53 & N/A \\
\hline EPA-SSS-10S & 35 & Undetermined \\
\hline EPA-SSS-12S & 46 & $\mathrm{~N} / \mathrm{A}$ \\
\hline
\end{tabular}


APPENDIX A

NPDES Outfalls by Category

\begin{tabular}{|c|c|c|}
\hline EPA Outfall Category & NPDES ID Number & Technical Area \\
\hline 001 & 001 & 57 \\
\hline $01 \mathrm{~A}$ & 001 & 3 \\
\hline $02 \mathrm{~A}$ & 007 & 16 \\
\hline $02 \mathrm{~A}$ & 129 & 21 \\
\hline $03 \mathrm{~A}$ & 009 & 3 \\
\hline $03 \mathrm{~A}$ & 020 & 2 \\
\hline $03 \mathrm{~A}$ & 021 & 3 \\
\hline $03 \mathrm{~A}$ & 022 & 3 \\
\hline $03 \mathrm{~A}$ & 023 & 3 \\
\hline $03 \mathrm{~A}$ & 024 & 3 \\
\hline $03 \mathrm{~A}$ & 025 & 3 \\
\hline $03 \mathrm{~A}$ & 027 & 3 \\
\hline $03 \mathrm{~A}$ & 028 & 15 \\
\hline $03 \mathrm{~A}$ & 031 & 21 \\
\hline $03 \mathrm{~A}$ & 032 & 21 \\
\hline $03 \mathrm{~A}$ & 034 & 21 \\
\hline $03 \mathrm{~A}$ & 035 & 21 \\
\hline $03 \mathrm{~A}$ & 036 & 21 \\
\hline $03 \mathrm{~A}$ & 037 & 21 \\
\hline $03 \mathrm{~A}$ & 038 & 33 \\
\hline $03 \mathrm{~A}$ & 040 & 43 \\
\hline $03 \mathrm{~A}$ & 042 & 46 \\
\hline $03 \mathrm{~A}$ & 043 & 46 \\
\hline $03 \mathrm{~A}$ & 045 & 48 \\
\hline $03 \mathrm{~A}$ & 047 & 53 \\
\hline $03 \mathrm{~A}$ & 048 & 53 \\
\hline $03 \mathrm{~A}$ & 049 & 53 \\
\hline $03 \mathrm{~A}$ & 060 & 16 \\
\hline $03 \mathrm{~A}$ & 098 & 59 \\
\hline $03 \mathrm{~A}$ & 113 & 53 \\
\hline $03 \mathrm{~A}$ & 114 & 53 \\
\hline $03 \mathrm{~A}$ & 124 & 46 \\
\hline $03 \mathrm{~A}$ & 125 & 53 \\
\hline $03 \mathrm{~A}$ & 130 & 11 \\
\hline $03 \mathrm{~A}$ & 136 & 46 \\
\hline $03 \mathrm{~A}$ & 145 & 53 \\
\hline $03 \mathrm{~A}$ & 146 & 53 \\
\hline $03 \mathrm{~A}$ & 148 & 3 \\
\hline $03 \mathrm{~A}$ & 158 & 21 \\
\hline
\end{tabular}




\begin{tabular}{|c|c|c|}
\hline EPA Outfall Category & NPDES ID Number & Technical Area \\
\hline $03 \mathrm{~A}$ & 160 & 35 \\
\hline $03 \mathrm{~A}$ & 181 & 55 \\
\hline $04 \mathrm{~A}$ & 013 & 46 \\
\hline $04 \mathrm{~A}$ & 014 & 46 \\
\hline $04 \mathrm{~A}$ & 016 & 48 \\
\hline $04 \mathrm{~A}$ & 018 & 46 \\
\hline $04 \mathrm{~A}$ & 070 & 16 \\
\hline $04 \mathrm{~A}$ & 083 & 16 \\
\hline $04 \mathrm{~A}$ & 091 & 16 \\
\hline $04 \mathrm{~A}$ & 092 & 16 \\
\hline $04 \mathrm{~A}$ & 093 & 15 \\
\hline $04 \mathrm{~A}$ & 094 & 3 \\
\hline $04 \mathrm{~A}$ & 101 & 40 \\
\hline $04 \mathrm{~A}$ & 102 & 15 \\
\hline $04 \mathrm{~A}$ & 103 & 15 \\
\hline $04 \mathrm{~A}$ & 109 & 3 \\
\hline $04 \mathrm{~A}$ & 115 & 8 \\
\hline $04 \mathrm{~A}$ & 117 & 46 \\
\hline $04 \mathrm{~A}$ & 118 & 0 \\
\hline $04 \mathrm{~A}$ & 126 & 48 \\
\hline $04 \mathrm{~A}$ & 127 & 35 \\
\hline $04 \mathrm{~A}$ & 131 & 48 \\
\hline $04 \mathrm{~A}$ & 133 & 53 \\
\hline $04 \mathrm{~A}$ & 135 & 53 \\
\hline $04 \mathrm{~A}$ & 137 & 48 \\
\hline $04 \mathrm{~A}$ & 139 & 15 \\
\hline $04 \mathrm{~A}$ & 140 & 3 \\
\hline $04 \mathrm{~A}$ & 141 & 39 \\
\hline $04 \mathrm{~A}$ & 142 & 21 \\
\hline $04 \mathrm{~A}$ & 143 & 15 \\
\hline $04 \mathrm{~A}$ & 147 & 33 \\
\hline $04 \mathrm{~A}$ & 151 & 3 \\
\hline $04 \mathrm{~A}$ & 152 & 48 \\
\hline $04 \mathrm{~A}$ & 153 & 48 \\
\hline $04 \mathrm{~A}$ & 155 & 9 \\
\hline $04 \mathrm{~A}$ & 156 & 39 \\
\hline $04 \mathrm{~A}$ & 157 & 16 \\
\hline $04 \mathrm{~A}$ & 161 & 0 \\
\hline $04 \mathrm{~A}$ & 163 & 0 \\
\hline $04 \mathrm{~A}$ & 164 & 0 \\
\hline $04 \mathrm{~A}$ & 165 & 0 \\
\hline $04 \mathrm{~A}$ & 166 & 0 \\
\hline
\end{tabular}




\begin{tabular}{|c|c|c|}
\hline EPA Outfall Category & NPDES ID Number & Technical Area \\
\hline $04 \mathrm{~A}$ & 167 & 0 \\
\hline $04 \mathrm{~A}$ & 168 & 0 \\
\hline $04 \mathrm{~A}$ & 169 & 0 \\
\hline $04 \mathrm{~A}$ & 170 & 0 \\
\hline $04 \mathrm{~A}$ & 171 & 0 \\
\hline $04 \mathrm{~A}$ & 172 & 0 \\
\hline $04 \mathrm{~A}$ & 173 & 0 \\
\hline $04 \mathrm{~A}$ & 174 & 0 \\
\hline $04 \mathrm{~A}$ & 175 & 0 \\
\hline $04 \mathrm{~A}$ & 176 & 0 \\
\hline $04 \mathrm{~A}$ & 177 & 0 \\
\hline $04 \mathrm{~A}$ & 178 & 0 \\
\hline $04 \mathrm{~A}$ & 179 & 0 \\
\hline $04 \mathrm{~A}$ & 182 & 21 \\
\hline $05 \mathrm{~A}$ & 052 & 16 \\
\hline $05 \mathrm{~A}$ & 053 & 16 \\
\hline $05 \mathrm{~A}$ & 054 & 16 \\
\hline $05 \mathrm{~A}$ & 055 & 16 \\
\hline $05 \mathrm{~A}$ & 056 & 16 \\
\hline $05 \mathrm{~A}$ & 057 & 16 \\
\hline $05 \mathrm{~A}$ & 058 & 16 \\
\hline $05 \mathrm{~A}$ & 061 & 16 \\
\hline $05 \mathrm{~A}$ & 062 & 16 \\
\hline $05 \mathrm{~A}$ & 063 & 16 \\
\hline $05 \mathrm{~A}$ & 066 & 9 \\
\hline $05 \mathrm{~A}$ & 067 & 9 \\
\hline $05 \mathrm{~A}$ & 068 & 9 \\
\hline $05 \mathrm{~A}$ & 069 & 11 \\
\hline $05 \mathrm{~A}$ & 071 & 16 \\
\hline $05 \mathrm{~A}$ & 072 & 16 \\
\hline $05 \mathrm{~A}$ & 096 & 11 \\
\hline $05 \mathrm{~A}$ & 097 & 11 \\
\hline $05 \mathrm{~A}$ & 149 & 16 \\
\hline $05 \mathrm{~A}$ & 154 & 40 \\
\hline $05 \mathrm{~A}$ & 159 & 16 \\
\hline $06 \mathrm{~A}$ & 073 & 16 \\
\hline $06 \mathrm{~A}$ & 074 & 8 \\
\hline $06 \mathrm{~A}$ & 075 & 8 \\
\hline $06 \mathrm{~A}$ & 078 & 22 \\
\hline $06 \mathrm{~A}$ & 079 & 40 \\
\hline $06 \mathrm{~A}$ & 080 & 40 \\
\hline $06 \mathrm{~A}$ & 081 & 40 \\
\hline
\end{tabular}




\begin{tabular}{|c|c|c|}
\hline EPA Outfall Category & NPDES ID Number & Technical Area \\
\hline $06 \mathrm{~A}$ & 082 & 40 \\
\hline $06 \mathrm{~A}$ & 099 & 40 \\
\hline $06 \mathrm{~A}$ & 100 & 40 \\
\hline $06 \mathrm{~A}$ & 106 & 36 \\
\hline $06 \mathrm{~A}$ & 123 & 15 \\
\hline $06 \mathrm{~A}$ & 132 & 35 \\
\hline 051 & 051 & 50 \\
\hline 128 & 128 & 22 \\
\hline SSS & $01 \mathrm{~S}$ & 3 \\
\hline SSS & $02 \mathrm{~S}$ & 9 \\
\hline SSS & $03 \mathrm{~S}$ & 16 \\
\hline SSS & $04 \mathrm{~S}$ & 18 \\
\hline SSS & $05 \mathrm{~S}$ & 21 \\
\hline SSS & $06 \mathrm{~S}$ & 41 \\
\hline SSS & $07 \mathrm{~S}$ & 46 \\
\hline SSS & $09 \mathrm{~S}$ & 53 \\
\hline SSS & $10 \mathrm{~S}$ & 35 \\
\hline SSS & $12 \mathrm{~S}$ & 46 \\
\hline
\end{tabular}


APPENDIX $B$

NPDES Outfalls by Technical Area

\begin{tabular}{|c|c|c|}
\hline Technical Area & EPA Outfall Category & NPDES ID Number \\
\hline 0 & $04 \mathrm{~A}$ & 118 \\
\hline 0 & $04 \mathrm{~A}$ & 161 \\
\hline 0 & $04 \mathrm{~A}$ & 163 \\
\hline 0 & $04 \mathrm{~A}$ & 164 \\
\hline 0 & $04 \mathrm{~A}$ & 165 \\
\hline 0 & $04 \mathrm{~A}$ & 166 \\
\hline 0 & $04 \mathrm{~A}$ & 167 \\
\hline 0 & $04 \mathrm{~A}$ & 168 \\
\hline 0 & $04 \mathrm{~A}$ & 169 \\
\hline 0 & $04 \mathrm{~A}$ & 170 \\
\hline 0 & $04 \mathrm{~A}$ & 171 \\
\hline 0 & $04 \mathrm{~A}$ & 172 \\
\hline 0 & $04 \mathrm{~A}$ & 173 \\
\hline 0 & $04 \mathrm{~A}$ & 174 \\
\hline 0 & $04 \mathrm{~A}$ & 175 \\
\hline 0 & $04 \mathrm{~A}$ & 176 \\
\hline 0 & $04 \mathrm{~A}$ & 177 \\
\hline 0 & $04 \mathrm{~A}$ & 178 \\
\hline 0 & $04 \mathrm{~A}$ & 179 \\
\hline 2 & $03 \mathrm{~A}$ & 020 \\
\hline 3 & $01 \mathrm{~A}$ & 001 \\
\hline 3 & $03 \mathrm{~A}$ & 009 \\
\hline 3 & $03 \mathrm{~A}$ & 021 \\
\hline 3 & $03 \mathrm{~A}$ & 022 \\
\hline 3 & $03 \mathrm{~A}$ & 023 \\
\hline 3 & $03 \mathrm{~A}$ & 024 \\
\hline 3 & $03 \mathrm{~A}$ & 025 \\
\hline 3 & $03 \mathrm{~A}$ & 027 \\
\hline 3 & $03 \mathrm{~A}$ & 148 \\
\hline 3 & $04 \mathrm{~A}$ & 094 \\
\hline 3 & $04 \mathrm{~A}$ & 109 \\
\hline 3 & $04 \mathrm{~A}$ & 140 \\
\hline 3 & $04 \mathrm{~A}$ & 151 \\
\hline 3 & SSS & $01 \mathrm{~S}$ \\
\hline 8 & $04 \mathrm{~A}$ & 115 \\
\hline 8 & $06 \mathrm{~A}$ & 074 \\
\hline 8 & $06 \mathrm{~A}$ & 075 \\
\hline 9 & $04 \mathrm{~A}$ & 155 \\
\hline 9 & $05 \mathrm{~A}$ & 066 \\
\hline
\end{tabular}




\begin{tabular}{|c|c|c|}
\hline Technical Area & EPA Outfall Category & NPDES ID Number \\
\hline 9 & $05 \mathrm{~A}$ & 067 \\
\hline 9 & $05 \mathrm{~A}$ & 068 \\
\hline 9 & SSS & $02 \mathrm{~S}$ \\
\hline 11 & $03 \mathrm{~A}$ & 130 \\
\hline 11 & $05 \mathrm{~A}$ & 069 \\
\hline 11 & $05 \mathrm{~A}$ & 096 \\
\hline 11 & $05 \mathrm{~A}$ & 097 \\
\hline 15 & $03 \mathrm{~A}$ & 028 \\
\hline 15 & $04 \mathrm{~A}$ & 093 \\
\hline 15 & $04 \mathrm{~A}$ & 102 \\
\hline 15 & $04 \mathrm{~A}$ & 103 \\
\hline 15 & $04 \mathrm{~A}$ & 139 \\
\hline 15 & $04 \mathrm{~A}$ & 143 \\
\hline 15 & $06 \mathrm{~A}$ & 123 \\
\hline 16 & $02 \mathrm{~A}$ & 007 \\
\hline 16 & $03 \mathrm{~A}$ & 060 \\
\hline 16 & $04 \mathrm{~A}$ & 070 \\
\hline 16 & $04 \mathrm{~A}$ & 083 \\
\hline 16 & $04 \mathrm{~A}$ & 091 \\
\hline 16 & $04 \mathrm{~A}$ & 092 \\
\hline 16 & $04 \mathrm{~A}$ & 157 \\
\hline 16 & $05 \mathrm{~A}$ & 052 \\
\hline 16 & $05 \mathrm{~A}$ & 053 \\
\hline 16 & $05 \mathrm{~A}$ & 054 \\
\hline 16 & $05 \mathrm{~A}$ & 055 \\
\hline 16 & $05 \mathrm{~A}$ & 056 \\
\hline 16 & $05 \mathrm{~A}$ & 057 \\
\hline 16 & $05 \mathrm{~A}$ & 058 \\
\hline 16 & $05 \mathrm{~A}$ & 061 \\
\hline 16 & $05 \mathrm{~A}$ & 062 \\
\hline 16 & $05 \mathrm{~A}$ & 063 \\
\hline 16 & $05 \mathrm{~A}$ & 071 \\
\hline 16 & $05 \mathrm{~A}$ & 072 \\
\hline 16 & $05 \mathrm{~A}$ & 149 \\
\hline 16 & $05 \mathrm{~A}$ & 159 \\
\hline 16 & $06 \mathrm{~A}$ & 073 \\
\hline 16 & SSS & $03 \mathrm{~S}$ \\
\hline 18 & SSS & $04 \mathrm{~S}$ \\
\hline 21 & $02 \mathrm{~A}$ & 129 \\
\hline 21 & $03 \mathrm{~A}$ & 031 \\
\hline 21 & $03 \mathrm{~A}$ & 032 \\
\hline 21 & $03 \mathrm{~A}$ & 034 \\
\hline
\end{tabular}




\begin{tabular}{|c|c|c|}
\hline Technical Area & EPA Outfall Category & NPDES ID Number \\
\hline 21 & $03 \mathrm{~A}$ & 035 \\
\hline 21 & $03 \mathrm{~A}$ & 036 \\
\hline 21 & $03 \mathrm{~A}$ & 037 \\
\hline 21 & $03 \mathrm{~A}$ & 158 \\
\hline 21 & $04 \mathrm{~A}$ & 142 \\
\hline 21 & $04 \mathrm{~A}$ & 182 \\
\hline 21 & SSS & $05 \mathrm{~S}$ \\
\hline 22 & $06 \mathrm{~A}$ & 078 \\
\hline 22 & 128 & 128 \\
\hline 33 & $03 \mathrm{~A}$ & 038 \\
\hline 33 & $04 \mathrm{~A}$ & 147 \\
\hline 35 & $03 \mathrm{~A}$ & 160 \\
\hline 35 & $04 \mathrm{~A}$ & 127 \\
\hline 35 & $06 \mathrm{~A}$ & 132 \\
\hline 35 & SSS & $10 \mathrm{~S}$ \\
\hline 36 & $06 \mathrm{~A}$ & 106 \\
\hline 39 & $04 \mathrm{~A}$ & 141 \\
\hline 39 & $04 \mathrm{~A}$ & 156 \\
\hline 40 & $04 \mathrm{~A}$ & 101 \\
\hline 40 & $05 \mathrm{~A}$ & 154 \\
\hline 40 & $06 \mathrm{~A}$ & 079 \\
\hline 40 & $06 \mathrm{~A}$ & 080 \\
\hline 40 & $06 \mathrm{~A}$ & 081 \\
\hline 40 & $06 \mathrm{~A}$ & 082 \\
\hline 40 & $06 \mathrm{~A}$ & 099 \\
\hline 40 & $06 \mathrm{~A}$ & 100 \\
\hline 41 & SSS & $06 \mathrm{~S}$ \\
\hline 43 & $03 \mathrm{~A}$ & 040 \\
\hline 46 & SSS & $07 \mathrm{~S}$ \\
\hline 46 & SSS & $12 \mathrm{~S}$ \\
\hline 46 & $03 \mathrm{~A}$ & 042 \\
\hline 46 & $03 \mathrm{~A}$ & 043 \\
\hline 46 & $03 \mathrm{~A}$ & 124 \\
\hline 46 & $03 \mathrm{~A}$ & 136 \\
\hline 46 & $04 \mathrm{~A}$ & 013 \\
\hline 46 & $04 \mathrm{~A}$ & 014 \\
\hline 46 & $04 \mathrm{~A}$ & 018 \\
\hline 46 & $04 \mathrm{~A}$ & 117 \\
\hline 48 & $03 \mathrm{~A}$ & 045 \\
\hline 48 & $04 \mathrm{~A}$ & 016 \\
\hline 48 & $04 \mathrm{~A}$ & 126 \\
\hline 48 & $04 \mathrm{~A}$ & 131 \\
\hline
\end{tabular}




\begin{tabular}{|c|c|c|}
\hline Technical Area & EPA Outfall Category & NPDES ID Number \\
\hline 48 & $04 \mathrm{~A}$ & 137 \\
\hline 48 & $04 \mathrm{~A}$ & 152 \\
\hline 48 & $04 \mathrm{~A}$ & 153 \\
\hline 50 & 051 & 051 \\
\hline 53 & $03 \mathrm{~A}$ & 047 \\
\hline 53 & $03 \mathrm{~A}$ & 048 \\
\hline 53 & $03 \mathrm{~A}$ & 049 \\
\hline 53 & $03 \mathrm{~A}$ & 113 \\
\hline 53 & $03 \mathrm{~A}$ & 114 \\
\hline 53 & $03 \mathrm{~A}$ & 125 \\
\hline 53 & $03 \mathrm{~A}$ & 145 \\
\hline 53 & $03 \mathrm{~A}$ & 146 \\
\hline 53 & $04 \mathrm{~A}$ & 133 \\
\hline 53 & $04 \mathrm{~A}$ & 135 \\
\hline 53 & SSS & $09 \mathrm{~S}$ \\
\hline 55 & $03 \mathrm{~A}$ & 181 \\
\hline 57 & 001 & 001 \\
\hline 59 & $03 \mathrm{~A}$ & 098 \\
\hline
\end{tabular}

